\title{
Terminating Evaluation of Logic Programs with Finite Three-Valued Models
}

FABRIZIO RIGUZZI, University of Ferrara

TERRANCE SWIFT, CENTRIA - Universidade Nova de Lisboa

\begin{abstract}
As evaluation methods for logic programs have become more sophisticated, the classes of programs for which termination can be guaranteed have expanded. From the perspective of answer set programs that include function symbols, recent work has identified classes for which grounding routines can terminate either on the entire program [Calimeri et al. 2008] or on suitable queries [Baselice et al. 2009]. From the perspective of tabling, it has long been known that a tabling technique called subgoal abstraction provides good termination properties for definite programs [Tamaki and Sato 1986], and this result was recently extended to stratified programs via the class of bounded term-size programs [Riguzzi and Swift 2013]. However, rather than asking what class of programs terminate for a given evaluation method, it is natural to start with a class of programs that have finite models, and then determine whether given evaluation methods terminate for that class. In this paper we define the class of strongly bounded term-size programs and show both that this class is equivalent to programs with finite well-founded models, and that for normal programs it strictly includes the finitely ground programs of [Calimeri et al. 2008]. We then show that tabling extended with suitable forms of subgoal abstraction terminates on all queries to such programs with an asymptotic complexity equal to the best known. Furthermore, tabling with subgoal abstraction produces a residual program that can be sent to an answer set programming system. Finally, we describe the implementation of subgoal abstraction within the SLG-WAM of XSB and provide performance results.
\end{abstract}

Categories and Subject Descriptors: D.1.6 [Programming Techniques]: Logic Programming

General Terms: Algorithms, Languages, Performance, Theory

Additional Key Words and Phrases: Tabled Logic Programming, Termination

\section{INTRODUCTION}

The study of termination has proven a fruitful topic in logic programming. The majority of work has focussed on analyzing termination of definite programs under SLD resolution and its extensions, such as arithmetic (e.g., [Decorte et al. 1999; Voets and De Schreye 2011]). Another recent branch of work has focused on defining classes of disjunctive programs for which a model-preserving ground instantiation can be obtained in finite time, along with algorithms to produce these instantiations [Baselice et al. 2009; Calimeri et al. 2008]. A third branch of work has explored the termination properties of query evaluation for definite or normal programs under tabling [Verbaeten et al. 2001; Riguzzi and Swift 2013]. The study of termination for tabling is of particular importance as tabling has come to underly several research and commercial knowledge representation systems [Alferes et al. 2013; Yang et al. 2012; Grosof et al. 2012].

Author's addresses: F. Riguzzi, fabrizio.riguzzi@unife.it Dipartimento di Matematica e Informatica - University of Ferrara, Via Saragat 1, I-44122, Ferrara, Italy; T. Swift, tswift@cs.suysb.edu CENTRIA - Universidade Nova de Lisboa.

Permission to make digital or hard copies of part or all of this work for personal or classroom use is granted without fee provided that copies are not made or distributed for profit or commercial advantage and that copies show this notice on the first page or initial screen of a display along with the full citation. Copyrights for components of this work owned by others than ACM must be honored. Abstracting with credit is permitted. To copy otherwise, to republish, to post on servers, to redistribute to lists, or to use any component of this work in other works requires prior specific permission and/or a fee. Permissions may be requested from Publications Dept., ACM, Inc., 2 Penn Plaza, Suite 701, New York, NY 10121-0701 USA, fax +1 (212) 869-0481, or permissions@acm.org.

(c) YYYY ACM 1529-3785/YYYY/01-ARTA $\$ 15.00$

DOI :http://dx.doi.org/10.1145/0000000.0000000 
Rather than starting with SLD resolution, with grounding techniques or with tabling, once could ask what evaluation methods terminate for programs with models that are finitely representable in some manner. Of course, a model (or interpretation) can be represented using sets of different elements - from one perspective a program itself is a set of rules representing its model - but for this paper we use the standard approach of representing models as sets of ground atoms ${ }^{1}$. The next question is what portion of a model needs to be represented. Let $P$ be a normal program with an infinite Herbrand base. A two-valued interpretaation, $\mathcal{I}_{P}$, of $P$ is arguably best represented by its set of true atoms $\left(\operatorname{true}\left(\mathcal{I}_{P}\right)\right)$, as reasoning can still be done in a complete manner on the false atoms $\left(\right.$ false $\left.\left(\mathcal{I}_{P}\right)\right)$ when the closed-world assumption is used. However, both tabling systems and grounders work with programs whose well-founded model may be three-valued, and if $\mathcal{I}_{P}$ is three-valued, at least two of its three truth assignments must be represented via finite sets. In this paper, we focus on programs that have three-valued models where both $\operatorname{true}\left(\mathcal{I}^{P}\right)$ and atoms whose truth assignment is undefined (undef $\left(\mathcal{I}^{P}\right)$ ) can be represented as finite sets of ground atoms. We term such finite models canonical.

Example 1.1. Consider the normal program $P_{\text {inf }}$ :

$p(s(X)) \leftarrow p(X)$.

$p(0)$.

$q(0)$.

$P_{\text {inf }}$ does not have a finite well-founded model (denoted $W F M^{P_{i n f}}$ ) as both true $\left(W F M^{P_{i n f}}\right)$ and false $\left(W F M^{P_{i n f}}\right)$ are infinite. However, the superficially similar program, $P_{\text {fin }}$ :

$p(X) \leftarrow p(f(X))$.

$p(0)$.

$q(0)$.

does have a (canonical) finite model, as $\operatorname{true}\left(W F M^{P_{i n f}}\right)$ and undef $\left(W F M^{P_{i n f}}\right)$ are both finite. Finally, the program $P_{\text {inf_undef }}$ :

$$
\begin{aligned}
& p(X) \quad \leftarrow p(f(X)) . \\
& p(0) . \\
& r(f(X)) \leftarrow \operatorname{not} r(X), \operatorname{not} r(f(X)) . \\
& q(0) .
\end{aligned}
$$

does not have a finite model, as undef $\left(W F M^{P_{\text {inf_undef }}}\right)$ is no longer finite.

This paper explores how programs that have canonical finite models relate to previous termination classes, and how such programs can be evaluated in a top-down manner. Specifically, the results of this paper are as follows.

- We extend the fixed-point definition of bounded term-size programs [Riguzzi and Swift 2013] to strongly bounded term-size programs, and show that this new notion coincides with the class of programs that have a canonical finite well-founded model. We then show that for programs that are both normal and safe, bounded term-size programs strictly include finitely ground programs [Calimeri et al. 2008].

\footnotetext{
${ }^{1}$ More general definitions are possible, allowing also the use of non-ground universally quantified atoms, or allowing non-ground atoms whose variables are subject to constraints over some domain.
} 
- We show that tabled SLG resolution, extended with subgoal abstraction, [Tamaki and Sato 1986; Riguzzi and Swift 2013] finitely terminates and correctly computes queries to safe, strongly bounded term-size programs. In addition, when depth-based abstraction functions are used, the abstract complexity of query evaluation equals the best complexity that is known ${ }^{2}$. As usual with SLG, the derived answers can be seen as a partially transformed program that preserves the stable model semantics, and so can be used by a grounder.

- We describe a publically available, engine-level implementation of subgoal abstraction that is sound and complete for safe, strongly bounded term-size programs, and provide performance results concerning this engine.

\section{BACKGROUND}

We recall those concepts of logic programming used in this paper. For a general treatment see [Lloyd 1987].

We assume a language $\mathcal{L}$ containing a finite set $\mathcal{F}$ of predicate and function symbols, and a countable set of program variables from the set $\mathcal{V}$. A term is either a variable (e.g., $X$ ), a function symbol of arity 0 (e.g., $c$ ) or a function symbol of arity $n$ applied to a tuple of $n$ terms (e.g., $f\left(t_{1}, \ldots, t_{n}\right)$ ). Symbols within a term may be represented through positions which are members of the set $\Pi$. A position in a term is either the empty string $\Lambda$ that reaches the root of the term, or the string $\pi . i$ that reaches the $i$ th child of the term reached by $\pi$, where $\pi$ is a position and $i$ an integer. For a term $t$ we denote the symbol at position $\pi$ in $t$ by $\left.t\right|_{\pi}$. For example, $\left.p(a, f(X))\right|_{2.1}=X$. We suppose that $\mathcal{L}$ also contains a countable set of variables $\hat{\mathcal{V}}$ called position variables that are of the form $X_{\pi}$, where $\pi$ is a position. A position variable is used in order to associate a given variable with a position of interest in a term.

An atom $A$ for a predicate symbol $p$ of arity $n$ is $p$ applied to a tuple of $n$ terms: $p\left(t_{1}, \ldots, t_{n}\right) ; \operatorname{pred}(A)$ indicates the predicate of the atom $A$. A literal is either an atom $A$ or the negation of an atom not $A$. A term, atom or literal is ground if it does not contain variables. A substitution $\theta$ is a set of pairs $V / s$ where $V$ is a variable and $s$ is a term. A substitution applied to a term/atom/literal $t$, indicated with $t \theta$, replace each variable $V$ in $t$ that appears in a pair $V / t$ in $\theta$ with $t$. An atom $A$ subsumes an atom $B$ if there is a substitution $\theta$ such that $A \theta=B$.

We assume that a program $P$ is defined over a language $\mathcal{L}$. The set of ground terms of a language $\mathcal{L}$ is called the Herbrand universe of $\mathcal{L}$ and is denoted by $\mathcal{H}_{\mathcal{L}}$, or as $\mathcal{H}_{P}$ if $\mathcal{L}$ consists of the predicate and function symbols in $P$. The set of ground atoms of a language $\mathcal{L}$ is called the Herbrand base and is denoted as $\mathcal{B}_{\mathcal{L}}$ or as $\mathcal{B}_{P}$. Two atoms are considered equal if they are variants of each other.

Throughout this paper we restrict our attention to normal programs, and to queries that are simply atoms. A normal program is a set of normal rules. We also assume a fixed strategy for selecting literals in a clause: without loss of generality we assume the selection strategy is left-to-right. In accordance with this strategy, a normal rule has the form

$$
r=H \leftarrow A_{1}, \ldots, A_{m}, \operatorname{not} A_{m+1}, \ldots, \operatorname{not} A_{n}
$$

where $A_{1}, \ldots, A_{n}$ are atoms. We say that a predicate symbol $p$ occurs positively (negatively) in $r$ if $p$ is the predicate symbol of an atom that occurs positively (negatively) in $r$. As notation, literals $(r)$ denotes the set of literals in the body of $r$ and head $(r)$ denotes the head $H$. A rule $r$ is safe if each variable in $r$ occurs in a positive literal

\footnotetext{
${ }^{2}$ The results of Section 4.6 are significantly more precise than previous complexity results for SLG, which showed that an evaluation required a number of operations that was polynomial in the size of a ground program.
} 
in the body of $r$, and a program is safe if all its rules are safe. For example, the rule $p(X, Y, Z) \leftarrow q(Y)$, not $r(Z)$. is not safe, because $X$ does not appear in the body and $Z$ appears only in a negative literal.

Given a program $P, \operatorname{Ground}(P)$ denotes the grounding of $P$; Facts $(P)$ denotes the set of rules with an empty body in $P$ and $\operatorname{Heads}(P)$ is the set of atoms in the head of some rule in $P$.

A two-valued interpretation $\mathcal{I}_{T}$ is a subset of $\mathcal{B}_{P} . \mathcal{I}_{T}$ is the set of true atoms. A threevalued interpretation $\mathcal{I}$ is a pair $\left\langle\mathcal{I}_{T} ; \mathcal{I}_{F}\right\rangle$ where $\mathcal{I}_{T}$ and $\mathcal{I}_{F}$ are subsets of $\mathcal{B}_{P}$ and represent respectively the set of true and false atoms. Alternatively, a three-value interpretation can be represented with a set of literals. The union of two three-valued interpretations $\left\langle\mathcal{I}_{T}, \mathcal{I}_{F}\right\rangle$ and $\left\langle\mathcal{J}_{T}, \mathcal{J}_{F}\right\rangle$ is defined as $\left\langle\mathcal{I}_{T}, \mathcal{I}_{F}\right\rangle \cup\left\langle\mathcal{J}_{T}, \mathcal{J}_{F}\right\rangle=\left\langle\mathcal{I}_{T} \cup \mathcal{J}_{T}, \mathcal{I}_{F} \cup \mathcal{J}_{F}\right\rangle$. A three-valued interpretation $\mathcal{I}$ is a subset of a three-valued interpretation $\mathcal{J}$ iff $\mathcal{I} \subseteq \mathcal{J}$ where $\mathcal{I}$ and $\mathcal{J}$ are represented as sets of literals.

To give a semantics to normal logic programs, we need to identify one or more interpretations as the "intended models" of the program, i.e., as the interpretations giving its meaning. Many semantics have been proposed for normal programs. Among these, the well-founded semantics [van Gelder et al. 1991] and the stable model semantics [Gelfond and Lifschitz 1988] are the most prominent.

\subsection{Well-Founded Semantics}

The well-founded semantics (WFS) assigns a three-valued model to a program, i.e., it identifies a three-valued interpretation as the meaning of the program. The WFS was given in [van Gelder et al. 1991] in terms of the least fixed point of an operator that is composed by two sub-operators, one computing consequences and the other computing unfounded sets. We give here the alternative definition of the WFS of [Przymusinski 1989] that is based on an iterated fixed point.

Definition 2.1. For a normal program $P$, sets $T r$ and $F a$ of ground atoms, and a 3 -valued interpretation $\mathcal{I}$ we define

$O p \operatorname{True} e_{\mathcal{I}}^{P}(\operatorname{Tr})=.\left\{A \mid A\right.$ is not true in $\mathcal{I}$; and there is a clause $B \leftarrow L_{1}, \ldots, L_{n}$ in $P$, a grounding substitution $\theta$ such that $A=B \theta$ and for every $1 \leq i \leq n$ either $L_{i} \theta$ is true in $\mathcal{I}$, or $\left.L_{i} \theta \in T r\right\}$;

$O p F a l s e_{\mathcal{I}}^{P}(F a)=.\left\{A \mid A\right.$ is not false in $\mathcal{I}$; and for every clause $B \leftarrow L_{1}, \ldots, L_{n}$ in $P$ and grounding substitution $\theta$ such that $A=B \theta$ there is some $i(1 \leq i \leq n)$ such that $L_{i} \theta$ is false in $\mathcal{I}$ or $\left.L_{i} \theta \in F a\right\}$.

[Przymusinski 1989] shows that $O p T r u e_{\mathcal{I}}^{P}$ and $O p F a l s e_{\mathcal{I}}^{P}$ are both monotonic, and defines $\mathcal{T}_{\mathcal{I}}^{P}$ as the least fixed point of $O p \operatorname{True} \mathcal{I}_{\mathcal{I}}^{P}(\emptyset)$ and $\mathcal{F}_{\mathcal{I}}^{P}$ as the greatest fixed point of $O p F a l s e_{\mathcal{I}}^{P}\left(\mathcal{B}_{P}\right)^{3}$. In words, the operator $\mathcal{T}_{\mathcal{I}}$ extends the interpretation $\mathcal{I}$ to add the new atomic facts that can be derived from $P$ knowing $\mathcal{I}$; while $\mathcal{F}_{\mathcal{I}}$ adds the new negations of atomic facts that can be shown false in $P$ by knowing $\mathcal{I}$ (via the uncovering of unfounded sets). An iterated fixed point operator builds up dynamic strata by constructing successive partial interpretations as follows. let

Definition 2.2 (Iterated Fixed Point and Dynamic Strata). For a normal program $P$

$$
\begin{aligned}
W F M_{0} & =\langle\emptyset ; \emptyset\rangle ; \\
W F M_{\alpha+1} & =W F M_{\alpha} \cup\left\langle\mathcal{T}_{W F M_{\alpha}} ; \mathcal{F}_{\left.W F M_{\alpha}\right\rangle ;}\right\rangle \\
W F M_{\alpha} & =\bigcup_{\beta<\alpha} W F M_{\beta}, \text { for limit ordinal } \alpha .
\end{aligned}
$$

\footnotetext{
${ }^{3}$ Below, we will sometimes omit the program $P$ in these operators when the context is clear.
} 
Let $W F M^{P}$ denote the fixed point interpretation $W F M_{\delta}$, where $\delta$ is the smallest (countable) ordinal such that both sets $\mathcal{T}_{W F M_{\delta}}$ and $\mathcal{F}_{W F M_{\delta}}$ are empty. We refer to $\delta$ as the depth of $P$. The stratum of atom $A$, is the least ordinal $\beta$ such that $A \in W F M_{\beta}$ (where $A$ may be either in the true or false component of $W F M_{\beta}$ ).

[Przymusinski 1989] shows that the iterated fixed point $W F M^{P}$ is in fact the wellfounded model, and that undefined atoms of the well-founded model do not belong to any stratum - i.e. they are not added to $W F M_{\delta}$ for any ordinal $\delta$. He called a program dynamically stratified if every atom belongs to a stratum. He also showed that a program has a two-valued well-founded model iff it is dynamically stratified, so that it is the weakest notion of stratification that is consistent with the well-founded semantics.

Example 2.3. Let us consider the program $P_{1}$

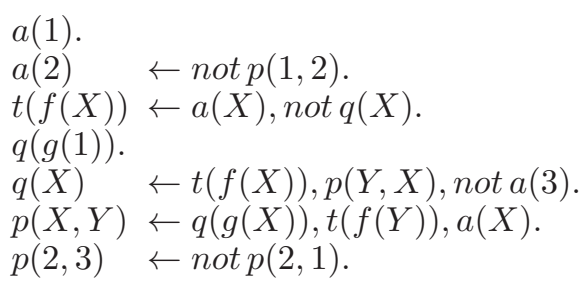

inspired by Example 1 of [Calimeri et al. 2008]. Its iterated fix point is

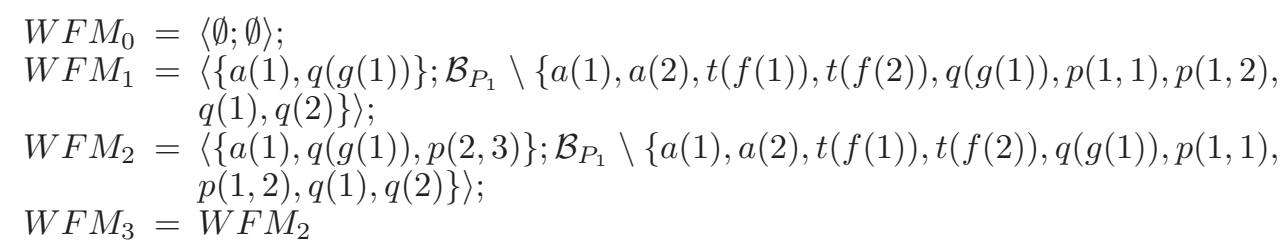

Thus the depth of $P_{1}$ is 3 and, for example, the stratum of $p(2,3)$ is 2 . The well-founded model of $P_{1}$ is given by

$$
\begin{aligned}
& \operatorname{true}\left(W F M^{P_{1}}\right)=\{a(1), q(g(1)), p(2,3)\} \\
& \text { undef }\left(W F M^{P_{1}}\right)=\{a(2), t(f(1)), t(f(2)), p(1,1), p(1,2), q(1), q(2)\}
\end{aligned}
$$

So $W F M^{P_{1}}$ is three-valued and $P_{1}$ is not dynamically stratified.

Given a normal program $P$, the atom dependency graph of $P$ is used to bound the search space of a derivation of a query $Q$ under the WFS.

Definition 2.4 (Atom Dependency Graph). Let $P$ be a normal program. Then the atom dependency graph of $P$ is a graph $(V, E)$ such that $V=\mathcal{B}_{P}$ and an edge $\left(v_{1}, v_{2}\right) \in E$ iff there is a grounding $r$ of a clause in $P$ such that $v_{1}=\operatorname{head}(r)$ and $v_{2}$ or $\neg v_{2} \in$ literals $(r)$

\subsection{Stable Model Semantics}

The stable model semantics [Gelfond and Lifschitz 1988] is the main alternative to the WFS. The stable models semantics associates zero, one or more two-valued models to a normal program.

Definition 2.5 (Reduction). Given a normal program $P$ and an interpretation $\mathcal{I}$, the reduction $\frac{P}{\mathcal{I}}$ of $P$ relative to $\mathcal{I}$ is obtained from $\operatorname{ground}(P)$ by deleting

(1) each rule that has a negative literal not $A$ such that $A \in \mathcal{I}$ 
(2) all negative literals in the body of the remaining rules.

Thus if $\mathcal{I}$ is a full two-valued interpretation, then $\frac{P}{\mathcal{I}}$ is a program without negation as failure and has a unique least Herbrand model $\operatorname{lhm}\left(\frac{P}{\mathcal{I}}\right)$.

Definition 2.6 (Stable Model). A two-valued interpretation $\mathcal{I}$ is a stable model or an answer set of a program $P$ if $\mathcal{I}=\operatorname{lhm}\left(\frac{P}{\mathcal{I}}\right)$.

The relationships between the WFS and the stable models semantics is given by the following two theorems [van Gelder et al. 1991].

THEOREM 2.7. If $P$ has a well-founded total model, then that model is the unique stable model.

THEOREM 2.8. The well-founded partial model of $P$ is a subset of every stable model of $P$ seen as a three-valued interpretation.

The problem of computing the answer sets of a program is called Answer Set Programming (ASP).

\subsection{Bounded term-size Programs}

Given the definitions of dynamic stratification, we are now in a position to define bounded term-size programs. Our definition extends that of [Riguzzi and Swift 2013] to use arbitrary norms on terms. that

Definition 2.9. A norm $N(\cdot)$ is a function from atoms to non-negative integers such

(1) $N(t)=0$ iff $t$ is a variable.

(2) $t$ subsumes $t^{\prime}$ implies $N(t) \leq N\left(t^{\prime}\right)$

A norm is finitary iff for a finite non-negative integer $k$, the cardinality of the set $\left\{t \mid t \in \mathcal{H}_{\mathcal{L}} \wedge N(t)<k\right\}$ is finite.

Definition 2.10 (Bounded Term-size Programs). Let $P$ a normal program, norm $(\cdot)$ a finitary norm, $\mathcal{I}$ a 3 -valued interpretation and $\operatorname{Tr} \subseteq \mathcal{B}_{P}$. Then an application of $\operatorname{OpTrue}_{\mathcal{I}}^{P}(\operatorname{Tr})$ (Definition 2.1) has the bounded term-size property if there is a integer $N$ such that $\operatorname{norm}(A)(=\operatorname{norm}(B \theta))$ is less than $N$ for all $A$ in $O p \operatorname{True} e_{\mathcal{I}}^{P}(\operatorname{Tr}) . P$ itself has the bounded term-size property if there is some $N$ for which every application of $O p T r u e_{\mathcal{I}}^{P}$ used to construct $W F M(P)$ has the bounded term-size property.

Note that $P_{i n f}$ from Example 1.1 does not have the bounded term-size property, but $P_{\text {fin }}$ and $P_{1}$ from Example 2.3 do. While determining whether a program $P$ is bounded term-size is clearly undecidable in general, $T_{f i n}$ shows that ground $(P)$ need not be finite if $P$ is bounded term-size.

2.3.1. Bounded Term-size Queries. Although bounded term-size programs have appealing properties, there are many interesting programs that are not bounded term-size. For instance, a program containing the Prolog predicate member $/ 2$ would not be bounded term-size, although as any Prolog programmer knows, a query to member $/ 2$ will terminate whenever the second argument of the query is ground. We capture this intuition with bounded term-size queries.

Definition 2.11 (Bounded Term-size Queries). Let $P$ be a normal program, and $Q$ an atomic query to $P$ (not necessarily ground). Then the atomic search space of $Q$ consists of the union of all ground instantiations of $Q$ in $\mathcal{B}_{P}$ together with all atoms reachable in the atom dependency graph of $P$ from any ground instantiation of $Q$. Let $P_{Q}=\{r \mid r$ grounding of a clause of $P$ and head $(r)$ is in the atomic search space of $Q\}$ 


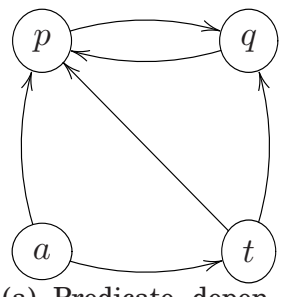

(a) Predicate dependency graph of $P_{1}$.

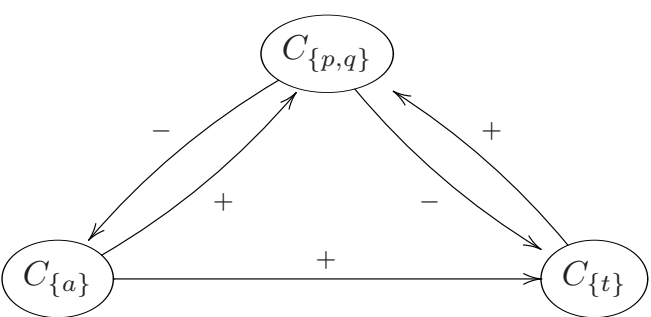

(b) Component graph of $P_{1}$.

Fig. 1. Graphs for $P_{1}$.

The query $Q$ is bounded term-size if $P_{Q}$ is a bounded term-size program.

In [Riguzzi and Swift 2013] it was shown that finitely recursive and bounded termsize programs are incompatible, but finitely recursive programs are a proper subclass of those programs for which all ground atomic queries are bounded term-size.

\subsection{Finitely Ground Programs}

Finitely ground programs were introduced in [Calimeri et al. 2008] as a class of logic programs with function symbols for which the set of ground instances of the rules that influence the computation of answer sets is finite.

The definition of finitely ground program relies on the notion of intelligent instantiation, which is a method to obtain a ground program from a program with variables so that no grounding of rules that matter for the computation of answer sets is excluded.

Intelligent instantiations and finitely ground programs were defined in [Calimeri et al. 2008] with respect to disjunctive normal programs. We here restrict these definitions to the case of non-disjunctive normal programs. Moreover, we do not distinguish between extensional and intensional predicates.

We first restate definitions from [Calimeri et al. 2008] that define dependency graphs for predicates and their components.

Definition 2.12. The predicate dependency graph $\mathcal{G}(P)$ of a program $P$ is a directed graph whose nodes are the predicates of $P$. There is an edge $\left(p_{2}, p_{1}\right)$ in $\mathcal{G}(P)$ if a rule for $p_{1}$ contains a positive literal for $p_{2}$ in its body ${ }^{4}$.

Example 2.13. Program $P_{1}$ of Example 2.3 has the predicate dependency graph shown in Figure 1(a).

Definition 2.14. Given a program $P$ and its predicate dependency graph $\mathcal{G}(P)$, the component graph of $\mathrm{P}, \mathcal{G}^{C}(P)$, is a directed labeled graph having a node for each maximal strongly connected component (SCC) of $\mathcal{G}(P) . \mathcal{G}^{C}(P)$ has an edge $\left(C_{2}, C_{1}\right)$ iff $C_{1} \neq C_{2}$ and there is a rule for some $p_{1} \in C_{1}$ such that $p_{2}$ occurs in its body. If $p_{2}$ occurs positively, the edge is labeled " + " and if $p_{2}$ occurs negatively, the edge is labeled '-' unless $\left(C_{2}, C_{1}\right)$ can be labeled as + .

An ordering can be defined over the component graph.

Definition 2.15. A path in $\mathcal{G}^{C}(P)$ is strong if all its edges are labeled + , and is weak otherwise. A component ordering $\mathcal{C}=\left(C_{0}, \ldots, C_{n}\right)$ for $P$ is a total ordering of the nodes in $\mathcal{G}^{C}(P)$ such that for any $C_{j}, C_{i}$ with $i<j$ then 1 ) there are no strong paths from $C_{j}$ to $C_{i}$ and 2) if there is a weak path from $C_{j}$ to $C_{i}$, then there is a weak path from $C_{i}$ to $C_{j}$.

\footnotetext{
${ }^{4}$ Note that this definition, unlike that of Definition 2.4 only creates edges for positive dependencies.
} 
Example 2.16. Program $P_{1}$ of Example 2.3 has three SCCs: $C_{\{a\}}$, containing only predicate $a, C_{\{t\}}$, containing only predicate $t$, and $C_{\{p, q\}}$, containing predicates $p$ and $q$.

The component graph for $P_{1}$ is shown in Figure 1(b). There are strong paths between $C_{\{a\}}$ and $C_{\{t\}}, C_{\{a\}}$ and $C_{\{p, q\}}, C_{\{t\}}$ and $C_{\{p, q\}}$ and weak paths between any couple of components. Thus the only component ordering is $\mathcal{C}=\left\{C_{\{a\}}, C_{\{t\}}, C_{\{p, q\}}\right\}$, so $C_{0}=C_{\{a\}}$, $C_{1}=C_{\{t\}}, C_{2}=C_{\{p, q\}}$.

Since each component corresponds to a set of predicates of $P$, the set of all components can be seen as a partition on the predicates of $P$. Moreover, each component $C_{i}$ corresponds with a module $P\left(C_{i}\right)$, a subprogram of $P$ containing all the rules with a predicate of $C_{i}$ in the head.

We now turn to definitions regarding program and rule instantiations. Supposing $\mathcal{T}$ is a set of atoms that are potentially true, we define a $\mathcal{T}$-restricted instance of a rule as one that is supported by $\mathcal{T}$.

Definition 2.17. Let $r$ be a rule and $\mathcal{T}$ a set of ground atoms. A $\mathcal{T}$-restricted instance $r^{\prime}$ of $r$ is a ground instance of $r$ such that if an atom $a$ occurs positively in the body of $r$ then $a \in \mathcal{T}$. The set of all $\mathcal{T}$-restricted instances of a program $P$ is denoted as $\operatorname{Inst}_{P}(\mathcal{T})$. then

Example 2.18. Given the program module $P_{1}\left(C_{\{t\}}\right)$ of program $P_{1}$ of Example 2.3,

$$
\begin{aligned}
& \text { Inst }_{P_{1}\left(C_{\{t\}}\right)}(\{a(1), a(2)\})=\{t(f(1)) \leftarrow a(1), \text { not } q(1) ., t(f(2)) \leftarrow a(2), \text { not } q(2)\} \\
& \text { Inst }_{P_{1}\left(C_{\{p, q\}}\right)}(\{a(1), a(2), t(f(1)), t(f(2)), q(g(1))\})=\{q(g(1)) \text {., } \\
& p(1,1) \leftarrow q(g(1)), t(f(1)), a(1) ., p(1,2) \leftarrow q(g(1)), t(f(2)), a(2) ., \\
& p(2,3) \leftarrow p(2,1) .\} \\
& \text { Inst }_{P_{1}\left(C_{\{p, q\}}(\{a(1), a(2), t(f(1)), t(f(2)), q(g(1)), p(1,1), p(1,2)\})=\{q(g(1)) .\right.} \text {. } \\
& p(1,1) \leftarrow q(g(1)), t(f(1)), a(1) ., p(1,2) \leftarrow q(g(1)), t(f(2)), a(2) . \\
& q(1) \leftarrow t(f(1)), p(1,1), \text { not } a(3) ., q(2) \leftarrow t(f(2)), p(1,2), \text { not } a(3) . \\
& p(2,3) \leftarrow p(2,1) .\}
\end{aligned}
$$

Assuming the program is evaluated from the bottom up using the component ordering, we can identify rule groundings that do not matter for the computation of answer sets and we can simplify the bodies of some others.

Definition 2.19. Given a program $P$ and a component ordering $\left(C_{0}, \ldots, C_{n}\right)$ for $P$, a set $\mathcal{S}_{j}$ of ground rules for component $C_{j}$ and a set of ground rules $\mathcal{R}$ for the components preceding $C_{j}$, the simplification of $\mathcal{S}_{j}$ with respect to $\mathcal{R}, \operatorname{Simpl}\left(\mathcal{S}_{j}, \mathcal{R}\right)$ is obtained from $\mathcal{S}_{j}$ by

(1) deleting each rule whose body contains some negative literal not $a$ such that $a \in$ $\operatorname{Facts}(\mathcal{R})$.

(2) eliminating from the remaining rules in $\mathcal{S}_{j}$ each literal $l$ such that

(a) $l$ is positive and $l \in F a c t s(\mathcal{R})$; or

(b) $l=\operatorname{not} a$, $\operatorname{pred}(a) \in C_{i}, i<j$, and $a \notin \operatorname{Heads}(\mathcal{R})$.

Example 2.20. Given program $P_{1}$ of Example 2.3, then

$$
\begin{aligned}
& \operatorname{Simpl}(\{a(1) ., a(2) \leftarrow \operatorname{not} p(1,2) .\}, \emptyset)= \\
&\{a(1),, a(2) \leftarrow \operatorname{not} p(1,2) .\} \\
& \operatorname{Simpl}(\{t(f(1)) \leftarrow a(1), \operatorname{not} q(1) ., t(f(2)) \leftarrow a(2), \operatorname{not} q(2) .\},\{a(1) ., a(2) \leftarrow \operatorname{not} p(1,2) .\})= \\
&\{t(f(1)) \leftarrow \operatorname{not} q(1), t(f(2)) \leftarrow a(2), \operatorname{not} q(2) .\} \\
& \operatorname{Simpl}(\{p(1,1) \leftarrow q(g(1)), t(f(1)), a(1) ., p(1,2) \leftarrow q(g(1)), t(f(2)), a(2) .,
\end{aligned}
$$




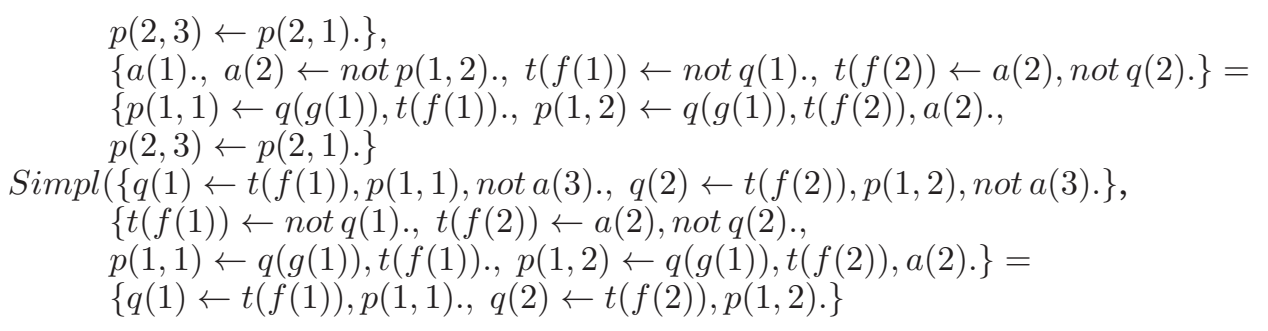

The operator $\phi$ defined below is used to select and simplify ground rules from a module $P\left(C_{j}\right)$ on the basis of a set of ground rules for preceding modules.

Definition 2.21. Let $P$ be a program with component ordering $\mathcal{C}=\left(C_{0}, \ldots, C_{n}\right)$, a component $C_{j}$, a set $\mathcal{X}_{j}$ of ground rules of $P\left(C_{j}\right)$, and a set $\mathcal{R}$ of ground rules of modules of components $C_{i}$ with $i<j$, let

$$
\phi_{C_{j}, \mathcal{R}}\left(\mathcal{X}_{j}\right)=\operatorname{Simpl}\left(\operatorname{Inst}_{P\left(C_{j}\right)}\left(\operatorname{Heads}\left(\mathcal{R} \cup \mathcal{X}_{j}\right)\right), \mathcal{R}\right)
$$

Since $\operatorname{Simpl}\left(\mathcal{S}_{j}, \mathcal{R}\right)$ is monotonic in its first argument, $\phi_{C_{j}, \mathcal{R}_{j}}$ is monotonic as well and has a least fixed point $l f p\left(\phi_{C_{j}, \mathcal{S}_{j-1}}(\emptyset)\right)$. We can consider $l f p\left(\phi_{C_{j}, \mathcal{S}_{j-1}}(\emptyset)\right)$ as an operator to be applied to components in order to drop many rules that do not influence answer set computation.

Definition 2.22. Let $P$ be a program and $\mathcal{C}=\left(C_{0}, \ldots, C_{n}\right)$ a component ordering for $P$. The intelligent instantiation $P^{\mathcal{C}}$ of $P$ for $\mathcal{C}$ is the last element $\mathcal{S}_{n}$ of the sequence

$$
\mathcal{S}_{0}=\operatorname{lfp}\left(\phi_{C_{0}, \emptyset}(\emptyset)\right) ; \mathcal{S}_{j}=\mathcal{S}_{j-1} \cup \operatorname{lfp}\left(\phi_{C_{j}, \mathcal{S}_{j-1}}(\emptyset)\right)
$$

Example 2.23. Given program $P_{1}$ of Example 2.3, then

$$
\begin{aligned}
& \phi_{C_{0}, \emptyset}^{1}(\emptyset)=\{a(1) ., a(2) \leftarrow \operatorname{not} p(1,2) .\} \\
& \left.\phi_{C_{0}, \emptyset}^{2}(\emptyset)=\phi_{C_{0}, \emptyset}^{1}(\emptyset)\right) \\
& \mathcal{S}_{0}=\operatorname{lfp}\left(\phi_{C_{0}, \emptyset}(\emptyset)\right) \stackrel{\mathcal{C}_{0}, \emptyset}{=}\{a(1) ., a(2) \leftarrow \operatorname{not} p(1,2) .\} \\
& \phi_{C_{1}, \mathcal{S}_{0}}^{1}(\emptyset)=\{t(f(1)) \leftarrow \operatorname{not} q(1) ., t(f(2)) \leftarrow a(2), \text { not } q(2) .\} \\
& \left.\phi_{C_{1}, \mathcal{S}_{0}}^{2}(\emptyset)=\phi_{C_{1}, \mathcal{S}_{0}}^{1}(\emptyset)\right) \\
& \mathcal{S}_{1}=\mathcal{S}_{0} \cup \operatorname{lfp}\left(\phi_{C_{1}, \mathcal{S}_{0}}(\emptyset)\right)=\{a(1) ., a(2) \leftarrow \operatorname{not} p(1,2) . \\
& t(f(1)) \leftarrow \operatorname{not} q(1) ., t(f(2)) \leftarrow a(2), \operatorname{not} q(2) .\} \\
& \phi_{C_{2}, \mathcal{S}_{1}}^{1}(\emptyset)=\{q(g(1)) \text {. } \\
& p(2,3) \leftarrow p(2,1) \cdot\} \\
& \phi_{C_{2}, \mathcal{S}_{1}}^{2}(\emptyset)=\{q(g(1)) ., p(1,1) \leftarrow t(f(1)) ., p(1,2) \leftarrow t(f(2)), a(2) ., \\
& p(2,3) \leftarrow p(2,1) .\} \\
& \phi_{C_{2}, \mathcal{S}_{1}}^{3}(\emptyset)=\{q(g(1)) ., p(1,1) \leftarrow t(f(1)) ., p(1,2) \leftarrow t(f(2)), a(2) . \\
& q(1) \leftarrow t(f(1)), p(1,1) ., q(2) \leftarrow t(f(1)), p(1,2) . \\
& p(2,3) \leftarrow p(2,1) .\} \\
& \left.\phi_{C_{2}, \mathcal{S}_{1}}^{4}(\emptyset)=\phi_{C_{2}, \mathcal{S}_{1}}^{3}(\emptyset)\right) \\
& \mathcal{S}_{2}=\mathcal{S}_{1} \cup \operatorname{lfp}\left(\phi_{C_{2}, \mathcal{S}_{1}}(\emptyset)\right)=\{a(1) ., a(2) \leftarrow \operatorname{not} p(1,2) . \\
& t(f(1)) \leftarrow \operatorname{not} q(1) ., t(f(2)) \leftarrow a(2), \operatorname{not} q(2) . \\
& q(g(1)) ., p(1,1) \leftarrow t(f(1)) ., p(1,2) \leftarrow t(f(2)), a(2) ., \\
& q(1) \leftarrow t(f(1)), p(1,1) ., q(2) \leftarrow t(f(1)), p(1,2) . \\
& p(2,3) \leftarrow p(2,1) .\}
\end{aligned}
$$


We are now ready to define finitely ground programs.

Definition 2.24. A program $P$ is finitely ground if its intelligent instantiation $P^{\mathcal{C}}$ is finite for all component orderings $\mathcal{C}$.

Example 2.25. Program $P_{1}$ of Example 2.3 is finitely ground as its intelligent instantiation is finite for the only component ordering.

Finitely ground programs enjoy the following property [Calimeri et al. 2008].

THEOREM 2.26. A finitely ground program has finitely many answer sets, and each of them is finite.

\section{STRONGLY BOUNDED TERM-SIZE PROGRAMS AND QUERIES}

A program that is bounded term-size may have an infinite number of undefined atoms. We define here strongly bounded term-size programs and queries.

Definition 3.1. A normal program $P$ is strongly bounded term-size iff it is bounded term-size, and in addition, undef $\left(W F M^{P}\right)$ is finite.

In [Riguzzi and Swift 2013] it was shown that for a normal program $P, P$ is bounded term-size iff $W F M^{P}$ has a finite number of true atoms. The following statement holds as a simple extension:

THEOREM 3.2. Let $P$ be a safe normal program. Then $W F M^{P}$ is has a canonical finite well-founded modele iff $P$ is strongly bounded term-size.

Proof. Theorem 1 from [Riguzzi and Swift 2013] states that if $P$ is a safe normal program, true $\left(W F M^{P}\right)$ is finite iff $P$ has the bounded term-size property. Definition 3.1 directly ensures that undef $\left(W F M^{P}\right)$ is also finite iff $P$ is strongly bounded term-size.

THEOREM 3.3. Let $P$ be a safe normal program. If $P$ is finitely ground then $P$ is strongly bounded term-size.

PROOF. By Theorem 2.8, an atom is true iff it is present in all answer sets. Again by Theorem 2.8, an atom is undefined iff it is present in some stable models and absent in others. Since $P$ is finitely ground, by Theorem 2.26 , it has finitely many answer sets and each of them is finite. Therefore the sets of true and undefined atoms are both finite and so $P$ is strongly bounded term-size.

Program $P_{1}$ of Example 2.3 is both finitely ground and strongly bounded term-size.

Example 3.4. The set of finitely ground program is a strict subset of the set of strongly bounded term-size programs. For example, the following program is strongly bounded term-size (and in fact, bounded term-size) but not finitely ground.

$$
\begin{array}{ll}
p(0) & \leftarrow \text { not } q . \\
p(f(X)) & \leftarrow p(X) . \\
q . & \\
q & \leftarrow \operatorname{not} p(1) . \\
q & \leftarrow p(1) .
\end{array}
$$

Its well-founded models is $\{q\}, \mathcal{B} \backslash\{q\}\rangle$. Its components are $C_{0}=\{p\}$ and $C_{1}=\{q\}$, it has a strong path from $\{p\}$ to $\{q\}$ and weak paths from $\{p\}$ to $\{q\}$ and vice-versa so its only component ordering is $\left\langle C_{0}, C_{1}\right\rangle$ and its intelligent instantiation is 


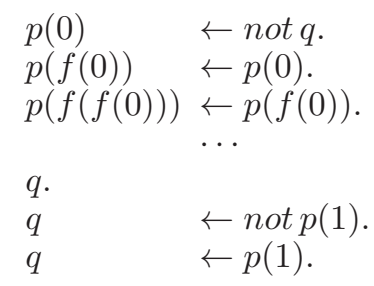

Theorem 3.3 together with Example 3.4 imply the following.

COROLlaRY 3.5. The class of safe programs that are strongly bounded term-size strictly includes the class of normal programs that are finitely ground.

Strongly Bounded Term-size queries are defined analogously to bounded term-size queries.

Definition 3.6 (Strongly Bounded Term-size Queries). Let $P$ be a normal program, and $Q$ an atomic query to $P$ (not necessarily ground). Then $Q$ is strongly bounded term-size if $P_{Q}$ is a strongly bounded term-size program.

\section{TABLED EVALUATION OF STRONGLY BOUNDED TERM-SIZE PROGRAMS}

In this section we present a tabled evaluation method that correctly evaluates strongly bounded term-size programs. Our approach is based on SLG evaluation [Chen and Warren 1996] which models well-founded computation for logic programs at an operational level, ensuring goal-directedness, termination and optimal complexity for a large class of programs. In this section we first present the main aspects of SLG informally through an example, and then briefly recall the definitions of SLG. Afterwards, we present our extension, SLG $_{S A}$ along with its properties.

\subsection{An Informal Review of SLG}

In the forest-of-trees model of SLG [Swift 1999], an evaluation is a possibly transfinite sequence of forests (sets) of trees that correspond to subgoals that have been encountered in an evaluation. The nodes in each tree contains sets of literals divided into those literals that have not been examined, and others that have been examined, but their resolution delayed (cf. Definition 4.2). The need to delay some literals arises for the following reason. Modern Prolog engines rely on a fixed order for selecting literals in a rule, e.g., always left-to-right. However, well-founded computations cannot be performed using a fixed-order literal selection function. Hence, in SLG, the DELAY operation may postpone evaluation of some literals, which may be later resolved through an operation called SIMPLIFICATION. In addition to supporting the operational behavior of Prolog, the use of delay and simplification supports the termination and complexity results discussed later in this section.

Example 4.1.

Consider the following program

$$
\begin{array}{ll}
r_{1}=p(b) . & \\
r_{2}=p(c) & \leftarrow \operatorname{not} p(a) . \\
r_{3}=p(X) & \leftarrow t(X, Y, Z), \operatorname{not} p(Y), \operatorname{not} p(Z) . \\
r_{4}=p(a) & \leftarrow p(b), p(a) . \\
r_{5}=t(a, a, b) . & \\
r_{6}=t(a, b, a) . &
\end{array}
$$


and query $p(c)$. The SLG forest at the end of this evaluation is shown in Figure 2 where each node is labeled with a number indicating the order in which it was created.

Nodes consist of either the symbol fail, or of a head representing the bindings made to an atomic subgoal and a body with a set of Delays, followed by the | symbol, followed by Goals that are still to be examined. The evaluation begins by creating a tree for the initial query with root $p(c) \leftarrow \mid p(c)$ in node 1 . Children of root nodes are created via the operation PROGRAM Clause RESOLUTION just as in the SLD resolution of Prolog. Accordingly, the evaluation uses rule $r_{2}$ to create node 2. The (only possible)

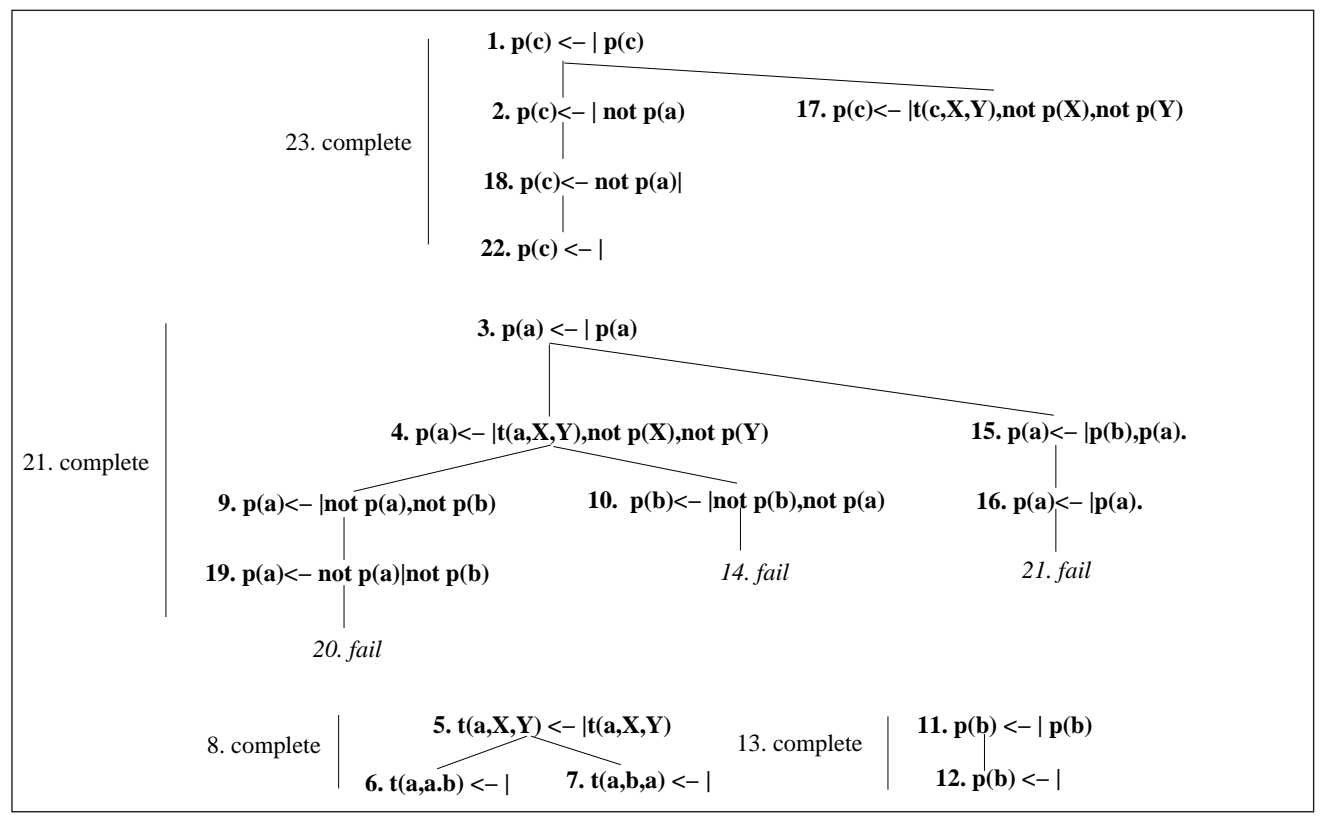

Fig. 2. Final forest for the query $p(c)$ to $P_{1}$.

literal not $p(a)$ in node 2 is selected. This literal has an underlying subgoal $p(a)$ that does not correspond to the root of any tree in the forest so far. Thus, the SLG operation NEW SubGoAL creates a new tree for $p(a)$ (node 3), whose child, node 4, is created by Program Clause Resolution using rule $r_{3}$. The NeW Subgoal operation is again used to create a new tree for the selected literal $t(a, X, Y)$ (node 5), and children nodes 6 and 7 are created by Program Clause Resolution from rules $r_{5}$ and $r_{6}$. These latter nodes have empty Goals and are termed answers; moreover, since they also have empty Delays, they are unconditional answers. ${ }^{5}$ Any atom in the ground instantiation of an unconditional answer is true in the well-founded model, cf. Theorem 4.13. The SLG operation POSITIVE RETURN is used to resolve the first of these answers against the selected literal of node 4 , producing node 9 . The selected literal of this latter node has $p(a)$ as its underlying subgoal, but there is already a tree for $p(a)$ in the forest and there are no answers for $p(a)$ to return. Since there is another unconditional answer for $t(a, X, Y)$ (node 7$)$, POSITIVE RETURN can be used to produce node 10 . The underlying

\footnotetext{
${ }^{5}$ In a practical program, a predicate defined by simple facts would not be evaluated using tabling, but rather would use SLD resolution as in Prolog.
} 
subgoal $p(b)$ is selected, the tree for $p(b)$ is created by NEW SUBGOAL (node 11), and it is eventually determined that the subgoal $p(b)$ has an unconditional answer (node 12); accordingly, using the NEGATION FAILURE operation, the failure node, node 14, is created. Then the computation, via PROGRAM Clause RESOLUTiON and rule $r_{4}$, produces another child for $p(a)$, node 15, and resolves away $p(b)$ creating node 16 . At this stage (up to node 16) the subgoal $p(a)$ is neither true, as no unconditional answers have been derived for it; nor false as one of its possible derivations, node 9, effectively has a loop through negation. However, it is possible to apply the DELAYING operation to the selected negative literal, by moving it from the Goals to the right of the | symbol into the Delays to the left of the | symbol. This DELAYING operation produces node 18, which is termed a conditional answer, as it has empty Goals but non-empty Delays ${ }^{6}$. DELAYING also produces node 19 whose new selected literal not $p(b)$ now fails (given the unconditional answer in node 12), producing the failure node 20. At this stage, all possible operations for non-answer nodes in $p(a)$ and the trees it depends on have been performed so that $p(a)$ may be completed (step 21). The completed subgoal $p(a)$ has no answers, and so is termed failed and is false in the well-founded model. This failed literal can be removed from the Delays of node 18 through the SIMPLIFICATION operation producing the unconditional answer node 22.

\subsection{SLG Evaluation}

SLG does not especially differ from other Prolog-like tabling formalisms in the case of programs that do not use default negation. However, as indicated in Example 4.1, for negation it introduces the concept of delaying literals in order to be able to find witnesses of failure anywhere in a rule, along with the concept of simplifying these delayed literals whenever their truth value becomes known.

An SLG evaluation proceeds by constructing a sequence of forests according to the set of SLG operations. Such forests, and the trees and nodes it contains are defined as follows:

Definition 4.2. A node has the form

$$
\text { AnswerTemplate } \leftarrow \text { Delays } \mid \text { Goals or fail. }
$$

In the first form, AnswerTemplate is an atom, while Delays and Goals are sequences of literals. The second form is called a failure node. An SLG tree $T$ has a root of the form $S \leftarrow \mid S$ for some atom $S$ : we call $S$ the root node for $T$ and $T$ the tree for $S$. An $S L G$ forest $\mathcal{F}$ is a set of SLG trees. A node $N$ is an answer when it is a leaf node for which Goals is empty. If the Delays of an answer is empty, it is termed an unconditional answer, otherwise, it is a conditional answer. A tree $T$ may be marked with the symbol complete.

The underlying subgoal of a literal $L$ is $L$ if $L$ is a positive literal; otherwise it is $S$ if $L=\operatorname{not} S$.

An SLG evaluation $\mathcal{E}$ of an atomic query $Q$ to a program $P$ is a sequence of forests. $\mathcal{E}$ starts with an initial forest containing the single node $Q \leftarrow \mid Q$ and creates the $n^{t h}$ forest in the sequence by applying an SLG operation if $n$ is a successor ordinal, or by taking the union of forests in previous sequences if $n$ is a limit ordinal. If no further operation is applicable, then the final forest for the evaluation of $Q$ has been reached. If there are selected non-ground negative literals in $\mathcal{F}$ then the evaluation is termed floundered. We introduce SLG operations incrementally, in Definitions 4.4, 4.6, and 4.9. Before we present the first set of operations, we present the definition of answer

${ }^{6}$ Choosing DELAYING in this order is not optimal and is made for purposes of illustrating the operations of SLG. This does not affect the result of the query itself since SLG is confluent [Chen and Warren 1996]. 
resolution, which differs from resolution in SLD in order to take account of Delays in conditional answers.

Definition 4.3. Let $N$ be a node $A \leftarrow D \mid L_{1}, \ldots, L_{n}$, where $n>0$. Let Ans $=A^{\prime} \leftarrow D^{\prime} \mid$ be an answer whose variables are disjoint from $N . N$ is $S L G$ resolvable with Ans if $\exists i$, $1 \leq i \leq n$, such that $L_{i}$ and $A^{\prime}$ are unifiable with a most general unifier $\theta$. The SLG resolvent of $N$ and Ans on $L_{i}$ has the form:

$$
\left(A \leftarrow D \mid L_{1}, \ldots, L_{i-1}, L_{i+1}, \ldots, L_{n}\right) \theta
$$

if $D^{\prime}$ is empty; otherwise the SLG resolvent has the form:

$$
\left(A \leftarrow D, L_{i} \mid L_{1}, \ldots, L_{i-1}, L_{i+1}, \ldots, L_{n}\right) \theta
$$

SLG resolution delays $L_{i}$ rather than propagating the answer's Delays, $D^{\prime}$, which means that $L_{i}$ in the Delays is only resolved once all of the delay literals of $D^{\prime}$ have become true or false. This is necessary, as shown in [Chen and Warren 1996], to ensure polynomial data complexity. ${ }^{7}$

Definition 4.4 (SLG Operations: 1 ). Let $P$ be a program and assume that a leftmost selection function is used to select a literal from the Goals in a node. Given a forest $\mathcal{F}_{n}$ of an SLG evaluation of $P, \mathcal{F}_{n+1}$ may be produced by one of the following operations.

(1) New SubGOAL: Let $\mathcal{F}_{n}$ contain a tree with non-root node

$$
N=\text { Ans } \leftarrow \text { Delays } \mid G, \text { Goals }
$$

where $S$ is the underlying subgoal of $G$. Assume $\mathcal{F}_{n}$ contains no tree with root $S$. Then add the tree $S \leftarrow \mid S$ to $\mathcal{F}_{n}$.

(2) Program Clause Resolution: Let $\mathcal{F}_{n}$ contain a tree with root node $N=S \leftarrow \mid S$ and $C$ be a rule Head $\leftarrow$ Body such that Head unifies with $S$ with mgu $\theta$. Assume that in $\mathcal{F}_{n}, N$ does not have a child $N_{\text {child }}=(S \leftarrow \mid$ Body $) \theta$. Then add $N_{\text {child }}$ as a child of $N$.

(3) Positive Return: Let $\mathcal{F}_{n}$ contain a tree with non-root node $N$ whose selected literal $S$ is positive. Let $A n s$ be an answer for $S$ in $\mathcal{F}_{n}$ and $N_{\text {child }}$ be the SLG resolvent of $N$ and Ans on $S$. Assume that in $\mathcal{F}_{n}, N$ does not have a child $N_{\text {child }}$. Then add $N_{\text {child }}$ as a child of $N$.

As illustrated in Example 4.1, NEW SUBGOAL creates a new tree in the forest $\mathcal{F}$ for a selected literal in the Goals of some (non-root) node in a tree in $\mathcal{F}$. Once a root node $N$ is created, the PROGRAM Clause RESOLUTION operation can create children for $N$, given the rules in the knowledge base. POSITIVE RETURN resolves positive literals in nodes with answers already in the forest, using SLG resolution according to Definition 4.3 .

If a sequence of SLG operations yields a (possibly intermediate) forest containing an unconditional answer, then this answer is considered to be true. Likewise, if no more operations are applicable to a set of trees, and none of them contains an unconditional answer, i.e., the set of literals associated to these trees is completely evaluated (see Definition 4.7), then we can interpret all these literals as false. Extending this correspondence, we associate an SLG forest with a partial interpretation. This interpretation is shown to correspond to the well-founded model. (cf. Theorem 4.13 below).

Definition 4.5. Let $\mathcal{F}$ be a SLG forest. Then the interpretation induced by $\mathcal{F}, \mathcal{I}_{\mathcal{F}}$, is the smallest set of literals such that:

\footnotetext{
${ }^{7}$ If Delays were propagated directly, then the Delays could effectively contain all derivations which could be exponentially many in the worst case.
} 
-A (ground) atom $A \in \mathcal{I}_{\mathcal{F}}$ iff $A$ is in the ground instantiation of some unconditional answer Ans $\leftarrow \mid$ in $\mathcal{F}$.

- A (ground) literal not $A \in \mathcal{I}_{\mathcal{F}}$ iff $A$ is in the ground instantiation of an atom whose tree in $\mathcal{F}$ is marked as complete, and $A$ is not in the ground instantiation of any answer in a tree in $\mathcal{F}$.

An atom $S$ is successful (resp. failed) in $\mathcal{F}$ if $S^{\prime}$ (resp. not $S^{\prime}$ ) is in $\mathcal{I}_{\mathcal{F}}$ for every $S^{\prime}$ in the ground instantiation of $S$. An atom not $S$ is successful (resp. failed) in $\mathcal{F}$ if not $S^{\prime}$ (resp. $\left.S^{\prime}\right)$ is in $\mathcal{I}_{\mathcal{F}}$ for every $S^{\prime}$ in the ground instantiation of $S$.

Given a three-valued interpretation $\mathcal{J}$ and forest $\mathcal{F}$, the restriction of $\mathcal{J}$ to $\mathcal{F},\left.\mathcal{J}\right|_{\mathcal{F}}$ is the interpretation such that $\operatorname{true}\left(\left.\mathcal{J}\right|_{\mathcal{F}}\right)\left(\operatorname{false}\left(\left.\mathcal{J}\right|_{\mathcal{F}}\right)\right)$ consists of those atoms in true $(\mathcal{J})$ $($ false $(\mathcal{J}))$ that are in the ground instantiation of some subgoal whose tree is in $\mathcal{F}$

Whenever an atom $A$ is successful, we can fail its default negation not $A$. If an atom $A$ is failed, then we can simplify away not $A$. Ground default negated literals that are neither failed nor successful may be delayed and later simplified. More precisely:

Definition 4.6 (SLG Operations: 2). Let $P$ be program and assume a selection function as in Definition 4.4. Given a forest $\mathcal{F}_{n}$ of an SLG evaluation of $P, \mathcal{F}_{n+1}$ may be produced by one of the following operations.

(4) Negative Return: Let $\mathcal{F}_{n}$ contain a tree with a leaf node, whose selected literal not $S$ is ground

$$
N=\text { Ans } \leftarrow \text { Delays } \mid \text { not } S, \text { Goals } .
$$

(a) Negation Success: If $S$ is failed in $\mathcal{F}_{n}$ then create a child for $N$ of the form: Ans $\leftarrow$ Delays $\mid$ Goals

(b) NEGATION FAILURE: If $S$ succeeds in $\mathcal{F}_{n}$, then create a child for $N$ of the form fail.

(5) DeLAYing: Let $\mathcal{F}_{n}$ contain a tree with leaf node

$$
N=\text { Ans } \leftarrow \text { Delays } \mid \text { not } S, \text { Goals }
$$

whose selected literal not $S$ is ground, but $S$ is neither successful nor failed in $\mathcal{F}_{n}$. Then create a child for $N$ of the form Ans $\leftarrow$ Delays, not $S \mid$ Goals.

(6) SIMPLIFICATION: Let $\mathcal{F}_{n}$ contain a tree with leaf node

$$
N=\text { Ans } \leftarrow \text { Delays } \mid
$$

and let $L \in$ Delays

(a) If $L$ is failed in $\mathcal{F}$ then create a child $f$ ail for $N$.

(b) If $L$ is successful in $\mathcal{F}$, then create a child Ans $\leftarrow$ Delays $^{\prime} \mid$ for $N$, where Delays $=$ Delays $\backslash\{L\}$.

SLG also includes an operation that marks a set of trees as complete if the corresponding set of subgoals is completely evaluated.

Definition 4.7. A set $\mathcal{S}$ of subgoals in a forest $\mathcal{F}$ is completely evaluated if at least one of the conditions holds for each $S \in \mathcal{S}$

(1) The tree for $S$ contains an answer $S \leftarrow$; or

(2) For each node $N$ in the tree for $S$ :

(a) The underlying subgoal of the selected literal of $N$ is marked as complete; or

(b) The underlying subgoal of the selected literal of $N$ is in $\mathcal{S}$ and there are no applicable New Subgoal, Program Clause Resolution, Positive Return (Definition 4.4), Negative Return or Delaying (Definition 4.6) operations for $N$. 
Once a set of subgoals is determined to be completely evaluated, a COMPLETION operation marks the trees for each subgoal (Definition 4.2). If condition 1 does not hold, condition 2(b) of the above definition prevents the COMPLETION operation from being applied to a tree from a set if certain other operations are applicable to the trees in the set. This notion of completion is incremental in the sense that once a set $\mathcal{S}$ of mutually dependent subgoals is fully evaluated, the derivation need not be concerned with the trees for $\mathcal{S}$ apart from any answers they contain. In an actual implementation resources for such trees are reclaimed.

In certain cases the propagation of delayed literals through SLG resolution (Definition 4.3) can lead to a set of unsupported answers - conditional answers that are false in the well founded model ${ }^{8}$. Intuitively, these answers, which have positive mutual dependencies through delay literals, correspond to an unfounded set, but their technical definition is based on the form of conditional answers.

Definition 4.8. Let $\mathcal{F}$ be an SLG forest, and $A$ be an atom that occurs in the head of some answer in a tree with root $S$. Then $A$ is supported in $\mathcal{F}$ if and only if:

(1) $S$ is not completely evaluated; or

(2) there exists an answer node $A^{\prime} \leftarrow$ Delays $\mid$ in $S$ such that $A^{\prime}$ subsumes $A$ and for every positive literal $L \in$ Delays, $L$ is supported in $\mathcal{F}$.

We are now able to characterize the last two SLG operations: one allows the completion of trees, and the other removes unsupported answers.

Definition 4.9 (SLG Operations: 3). Let $P$ be a program. Given a forest $\mathcal{F}_{n}$ of an SLG evaluation of $P, \mathcal{F}_{n+1}$ may also be produced by one of the following operations.

(8) Completion: Given a completely evaluated set $\mathcal{S}$ of subgoals (Definition 4.7), mark the trees for all subgoals in $\mathcal{S}$ as complete.

(9) Answer Completion: Given a set of unsupported atoms $\mathcal{U} \mathcal{A}$, create a failure node as a child for each answer whose head is in $\mathcal{U} \mathcal{A}$.

Each of the operations (1)-(9), in Definitions 4.4, 4.6 and 4.9, can be seen as a function that associates a forest with a new forest by adding a new tree, adding a new node to an existing tree, or marking a set of trees as complete.

\subsection{Extending SLG with Subgoal Abstractions}

An abstraction of a term $t$, denoted $a b s(t)$, may replace subterms of $t$ by position variables: formally, $a b s(t)$ is a term such that if $\left.a b s(t)\right|_{\pi} \in(\mathcal{F} \cup \mathcal{V})$, then $\left.a b s(t)\right|_{\pi}=\left.t\right|_{\pi}$. For instance $p\left(f\left(g\left(X_{1.1 .1}\right), X_{1.2}\right), X_{2}\right)$ is an abstraction of $p(f(g(a), X), X)$. It is easy to see that $a b s(t)$ subsumes $t$. An abstraction abs is finitary if the cardinality of $\left\{a b s(t) \mid t \in \mathcal{H}_{\mathcal{L}}\right\}$ is finite. Norms and abstractions may be applied to atoms by taking those atoms as terms.

Example 4.10. A depth norm, denoted $\operatorname{depth}(\cdot)$, is a norm that maps $t$ to the maximal depth of any position in $t$, where the depth of the outermost function symbol of $t$ is 1 and the depth of a position $\pi . i$ is the depth of $\pi$ plus 1 if $\left.t\right|_{\pi . i}$ is a not a position variable, and is the depth of $\pi$ otherwise. For a positive integer $k$, we define a depth- $k$ abstraction: an abstraction that maps a term $t$ to itself if depth $(t)$ is less than or equal to $k$, and to the maximal abstraction of $t$ with depth $k$ otherwise. It is easy to see that the maximal depth- $k$ abstraction of $t$ must be unique. Within the atom $A=p(a, f(b, g(c)))$ the depth of $c$ is 4 . The depth 3 abstraction of $A$ is $p\left(a, f\left(b, g\left(X_{2.2 .1}\right)\right)\right)$, and the depth 2

\footnotetext{
${ }^{8} \mathrm{As}$ an aside, we note that unsupported answers appear to be uncommon in evaluation strategies that minimize the use of delay, such as those used by XSB [Swift and Warren 2012].
} 
abstraction of $A$ is $p\left(a, f\left(X_{2.1}, X_{2.2}\right)\right)$. Both the depth norm and the family of depth- $k$ abstractions are finitary for any positive integer $k$. As a convention, we consider the identity function as a depth- $\omega$ abstraction.

Depth- $k$ abstractions are simple to understand and to implement. However the number of terms whose depth is less than $k$ may grow exponentially in many languages. Thus, other abstractions can be practically useful: such as those based on the size of a term, or those that weigh the occurrence of certain types of function symbols over others (e.g., weighing list symbols less than other function symbols). Finally, note that the identity function on terms is an abstraction function, but is not finitary.

The single extension to basic SLG needed to ensure finite evaluations for strongly bounded term-size programs is the addition of abstraction functions to the NEW SUBGOAL operation.

Definition 4.11. NEW SUbGOAL: Let $\mathcal{F}_{n}$ contain a tree with non-root node

$$
N=\text { Ans } \leftarrow \text { Delays } \mid G, \text { Goals }
$$

where $S$ is the underlying subgoal of $G$. Assume $\mathcal{F}_{n}$ contains no tree with root abs $(S)$. Then add the tree $a b s(S) \leftarrow \mid a b s(S)$ to $\mathcal{F}_{n}$.

We denote this extended version as SLG $_{S A}$ to distinguish it from previous versions in the literature.

Example 4.12. Consider the goal $p(1)$ to the program $P_{\text {fin }}$ from Example 1.1. If this goal is evaluated with basic SLG, an infinite number of goals will be created: $p(1)$, $p(f(1)), p(f(f(1)))$, and so on. However, if evaluated with a depth-3 abstraction function, only the first two of these goals together with $p\left(f\left(f\left(X_{1.1 .1}\right)\right)\right)$ would be created, neither of which would have any answers. Note that the technique of call subsumption, which is used by some tabling methods, would not help in the basic case where subgoal abstraction is not used, as none of the goals $p(1), p(f(1)), p(f(f(1))), \ldots$ subsume one another.

\subsection{Results}

The following theorem shows that SLG $_{S A}$ has the same correctness property as SLG, regardless of whether it is evaluating a query to a program that is strongly bounded term-size or not. Correctness does not require safety, and may involve transfinite evaluation.

THEOREM 4.13. Let $\mathcal{E}$ be an $S L G_{\mathrm{SA}}$ evaluation of a query $Q$ to a safe program $P$, Then $\mathcal{I}_{\mathcal{F}_{\text {fin }}}=\left.W F M(P)\right|_{\mathcal{F}_{\text {fin }}}$.

As stated below, SLGA terminates on any strongly bounded term-size program, although the use of subgoal abstraction on goals to rules with non-safe negation may introduce floundering.

THEOREM 4.14. Let $Q$ be a query to a strongly bounded term-size program P. Then any $S L G_{\mathrm{SA}}$ evaluation $\mathcal{E}$ of $Q$ that uses a finitary abstraction operation reaches a final forest $\mathcal{F}_{\text {fin }}$ after a finite number of steps. If $P$ is safe, then $\mathcal{F}_{\text {fin }}$ will not be floundered.

\section{5. $S L G_{S A}$ and Intelligent Instantiation}

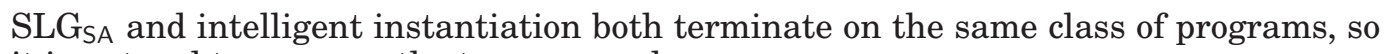
it is natural to compare the two approaches.

Example 4.15. Consider the program: 
$p(X, Y) \leftarrow t(X, Y, Z), \operatorname{not} p(Y, Z)$
$t(a, b, c)$

The intelligent instantiation of this program is the set of clauses

$$
\{t(a, b, c) ., p(a, b) \leftarrow \operatorname{not} p(b, c) .\}
$$

Note that because $p(b, c)$ and $p(a, b)$ are in the same component, not $p(b, c)$ cannot be removed from the body of the second clause. However, because SLG evaluates negation based on dynamic dependencies, the interpretation of the final forest for the top-level goal $p(X, Y)$ will assign $p(a, b)$ as true, and all other instantiations of $p(X, Y)$ as false.

In order to compare SLG SA $_{\text {A }}$ to intelligent instantiation more precisely, we need a framework to compare their results. First, since intelligent instantiation grounds an entire program while SLG SA $_{\text {A }}$ is uery-oriented, we introduce the notion of a grounding predicate to ensure that an entire program is reduced. Let $P$ be a safe normal program for which every predicate is tabled. Then the grounding predicate $P_{\text {grounding }}$ is defined by the set of rules:

$$
\begin{aligned}
& P_{\text {grounding }} \leftarrow \operatorname{pred}_{1}\left(\vec{X}_{1}\right) \\
& P_{\text {grounding }} \leftarrow \operatorname{pred}_{n}\left(\vec{X}_{n}\right)
\end{aligned}
$$

where for each predicate, pred $d_{i}$ occurring in $P, \operatorname{pred}_{i}\left(\vec{X}_{i}\right)$ is an atom of pred $d_{i}$ whose arguments consist solely of position variables.

Next, we specify a way to compare two ground instantiations of the same program. Let $r_{1}=$ Head $\leftarrow$ Body $y_{1}$ and $r_{2}=$ Head $\leftarrow$ Body $y_{2}$ be ground clauses: $r_{1}$ is at least as reduced than $r_{2}, r_{1} \geq_{\text {red }} r_{2}$, iff literals $\left(B o d y_{1}\right) \subseteq$ literals $\left(B o d y_{2}\right)$. Similarly, for ground programs $P_{1}, P_{2}, P_{1} \geq_{r e d} P_{2}$ iff for every rule $r_{1}$ in $P_{1}$ there is a rule $r_{2}$ in $P_{2} r_{1} \geq_{r e d} r_{2}$. Finally as notation, if $\mathcal{F}$ is an $\operatorname{SLG}_{\mathrm{SA}}$ forest, then answers $(\mathcal{F})$ is the set of answers in $\mathcal{F}$ taken as program clauses.

The following theorem indicates that, for finitely ground programs, SLGSA is at least as effective a grounder as intelligent instantiation. Its proof essentially follows from

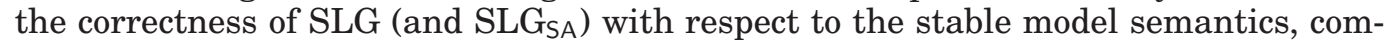
bined with Theorem 2.26 .

THEOREM 4.16. Let $P$ be a safe, finitely ground program $P$. Let $\mathcal{E}$ be a $S L G_{\mathrm{SA}}$ evaluation of a grounding predicate of $P$ whose final forest is $\mathcal{F}_{\text {fin }}$, and $P_{\text {tabled }}=$ $\operatorname{answers}\left(\mathcal{F}_{\text {fin }}\right)$.

(1) $\operatorname{ground}\left(P_{\text {tabled }}\right)$ is equal to $\frac{\operatorname{ground}(P)}{W F M(P)}$.

(2) Let $P_{i i}$ be the intelligent instantiation of $P$. Then ground $\left(P_{\text {tabled }}\right) \geq_{\text {red }} P_{i i}$.

\subsection{Complexity of SLG ${ }_{S A}$}

While the abstract complexity of query evaluation has been studied for SLG and its extensions (e.g., [Chen and Warren 1996; Alferes et al. 2013]), the results obtained are typically that evaluation of a ground query has polynomial complexity in the size of a given function-free program. Since SLG $_{S A}$ differs only from SLG in its NEW SUBGOAL operation, a similar result can be shown for SLG $_{S A}$, assuming proper conditions for $a b s(\cdot)$. However, such a result does not provide any insight into the behavior of $\mathrm{SLG}_{\mathrm{SA}}$ on strongly bounded term-size programs that contain function symbols: the very type of programs it is designed to address.

In previous approaches to complexity (e.g., [van Gelder 1989]) $P$ is a (finite) ground program without function symbols. Define size $(r)$ for a rule $r$ as one plus the number 
of body literals in $r$; size $(P)$ for a program $P$ is the sum of the size of each rule. Next, let atoms $(P)$ indicate the set of atoms appearing in $P$. Then the best currently known bound on worst case complexity for computing the well-founded semantics of an unrestricted normal program $P$ is size $(P) \times \mid$ atoms $(P) \mid$ [van Gelder 1989], and is shown by induction on the alternating fixed point computation of $P$.

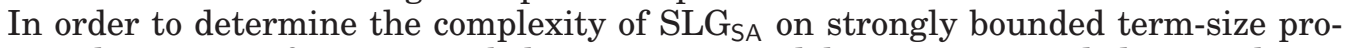
grams that contain function symbols, a new cost model $\mathcal{C}_{\text {function }}$ is needed, as neither $P$ nor $P_{Q}$ (Definition 3.6) need be finite. Accordingly, let $P$ be a ground strongly bounded term-size program with function symbols, and $Q$ a ground query. In the cost model $\mathcal{C}_{\text {function }}$, the size of a rule $r$ is defined as above: that is, one plus the number of body literals in $r$. Therefore size (.) does not consider the number of symbols or the depth of terms within an atom or literal, but treats size as a constant. By Theorem 4.14, an SLG $_{\mathrm{SA}}$ evaluation $\mathcal{E}$ of $Q$ against $P$ that uses a finitary abstraction function will produce a final forest $\mathcal{F}_{\text {fin }}$ after a finite number of steps, and $\mathcal{F}_{\text {fin }}$ will itself be finite. Given $\mathcal{E}$, we can construct the set of (ground) rules that were used in some PROGRAM Clause Resolution operation and denote this set as $P_{Q}(\mathcal{E})$. Since $P_{Q}$ is constructed from the atom dependency graph rather than from a dynamic computation, it is evident that $P_{Q}(\mathcal{E}) \subseteq P_{Q}$, and since $\mathcal{E}$ is finite, $P_{Q}(\mathcal{E})$ must always be finite. Next, define atoms $\left(\mathcal{F}_{\text {fin }}\right)$ as the set of atoms that occur as the head of some node in $\mathcal{F}_{\text {fin }}$. Note that this set may contain every atom in $P_{Q}$ plus those roots of trees that have been abstracted via a non-trivial application of the depth- $k$ abstraction function. However in this case, $\mid$ atoms $\left(\mathcal{F}_{f i n}\right) \mid$ is bounded by $2 \times\left|\operatorname{atoms}\left(P_{Q}\right)\right|$ if atoms $\left(P_{Q}\right)$ is finite. In addition, it is evident that atoms $\left(\mathcal{F}_{\text {fin }}\right)$ (the set of atoms occurring in any node in $\mathcal{F}_{\text {fin }}$ ) is finite, although atoms $\left(P_{Q}\right)$ may not be.

We state these observations formally.

LEMMA 4.17. Let $P$ be a ground strongly bounded term-size program and $Q$ a ground query. Let $\mathcal{E}$ be an $S L G_{\mathrm{SA}}$ evaluation of $Q$ against $P_{Q}$ that uses a finitary abstraction function, and let the final forest of $\mathcal{E}$ be $\mathcal{F}_{\text {fin. }}$. Then $P_{Q}(\mathcal{E})$ is finite and $P_{Q}(\mathcal{E}) \subseteq P_{Q}$. In addition atoms $\left(\mathcal{F}_{\text {fin }}\right)$ is finite, and if atoms $\left(P_{Q}\right)$ is also finite $\left|\operatorname{atoms}\left(\mathcal{F}_{\text {fin }}\right)\right| \leq 2 \times \mid\left(\operatorname{atoms}\left(P_{Q}\right) \mid\right.$.

The goal is thus to prove a complexity bound of $\operatorname{size}\left(P_{Q}(\mathcal{E})\right) \times\left|\operatorname{atoms}\left(\mathcal{F}_{\text {fin }}\right)\right|$ which is finite and at most size $(P) \times 2 \times \mid$ atoms $(P) \mid$. Towards this end, the following lemma bounds the number of nodes in the final forest of an evaluation. Its proof depends on showing that a ground program clause is used to produce a node in exactly one tree in a computation. This property holds under $\mathcal{C}_{\text {function }}$ for depth- $k$ abstractions, and remains open for arbitrary abstractions.

LEMMA 4.18. Let $P$ be a ground program, $Q$ a ground query, and $\mathcal{E}$ a terminating $S L G_{\mathrm{SA}}$ evaluation of $Q$ against $P$ that uses depth- $k$ abstraction. Then the number of nodes in the final forest $\mathcal{F}_{\text {fin }}$ is at most $\mathcal{O}\left(\operatorname{size}\left(P_{Q}(\mathcal{E})\right)\right)$.

As a next step in defining $\mathcal{C}_{\text {function }}$, we consider the cost of each SLGSA operation. First, since the scope of an abstraction function is an atom, the cost of applying an abstraction function is constant in $\mathcal{C}_{\text {function }}{ }^{9}$. Note that the NEW SUBGOAL operation creates a root node for a given atomic subgoal, and thus may be considered a constanttime operation. Similarly the Program Clause Resolution, Positive Return, Negative Return, Delaying, and SimplificAtion operations each affect one goal or delay literal and may also be considered constant-time. The COMPLETION operation, however, applies to a set of subgoals $\mathcal{S}$ in a forest $\mathcal{F}$ and its cost is proportional

\footnotetext{
${ }^{9}$ Of course a practical implementation of an abstraction function should have a low cost as a function of the actual size of an atom to which it is applied.
} 
to the cardinality of $\mathcal{S}$ : in the worst case this is $\mid$ atoms $(\mathcal{F}) \mid$. Similarly, the ANSwER COMPLETION operation must determine an unsupported set of answers and its worst case is $\operatorname{size}\left(P_{Q}(\mathcal{E})\right)$.

The cost model $\mathcal{C}_{\text {function }}$ thus consists of the definition of the size of a program that contains function symbols, along with constants for each individual SLGA operation.

THEOREM 4.19. Let $P$ be a ground program, $Q$ a ground query, and $\mathcal{E}$ a terminating $S L G_{S A}$ evaluation of $Q$ against $P$ that uses depth- $k$ abstraction, and with final forest $\mathcal{F}_{\text {fin }}$. Then under the cost model $\mathcal{C}_{\text {function }}$, the cost of $\mathcal{E}$ is $\mathcal{O}\left(\left|\operatorname{atoms}\left(\mathcal{F}_{\text {fin }}\right)\right| \times \operatorname{size}\left(P_{Q}(\mathcal{E})\right)\right)$.

\section{IMPLEMENTATION OF SUBGOAL ABSTRACTION}

Depth- $k$ subgoal abstraction is built into the XSB engine and can be invoked in Version 3.3.8 of XSB (xsb.sourceforge.net) in two ways. First, a default maximum depth can be set for tabled subgoals, along with an action to take if that depth is exceeded: both the depth and action are changeable by Prolog flags. The default action is abstract which gives the semantics discussed in previous sections, but can be set to error which causes a Prolog permission error to be thrown, or warning. In addition to setting it by a default flag, the subgoal depth can be set on a per-predicate basis by the directive:

$$
\therefore-\text { table }<\text { predspec }>\text { as subgoal_depth }(<n>), \ldots
$$

This depth declaration overrides the default, and subgoal_depth $(<n>)$ is a table property that can be combined with other properties such as incremental, thread-private, thread-shared, and so on ${ }^{10}$. In order to avoid the creation of floundering subgoals, XSB only abstracts positive literals; however, this limitation does not affect termination for bounded term-size programs that are safe, as the binding of each variable in a negative goal must have been produced as part of an answer to a positive subgoal. Within $\mathrm{XSB}$, abstraction is permitted on subgoals with attributed variables, which support constraint-based reasoning, as described below.

At a high level, the implementation of subgoal abstraction can be seen as a dynamically performed rewrite of a subgoal:

$$
\ldots, G \theta, \ldots \Rightarrow \ldots, a b s(G \theta), a b s(G \theta)=G \theta, \ldots
$$

i.e., the goal $G \theta$ is replaced by the depth- $k$ abstraction $a b s(G \theta)$ and $a b s(G \theta)$ is called; any answers returned for $a b s(G \theta)$ are unified with the original goal $G \theta$ - a step we term post-unification.

Example 5.1. As a concrete example, suppose the goal $p(X)$ was made to the program $P_{\text {sbts }}$

$$
\begin{aligned}
& p(1) \quad \leftarrow p(f(1)) . \\
& p(f(f(X))) \leftarrow q(X) . \\
& q(0) . \\
& q(1) .
\end{aligned}
$$

in an evaluation where depth-3 abstraction is used. The tabled subgoal $p(1)$ produces the subgoal $p(f(1))$ by PROGRAM Clause RESOLUTION against the clause $p(X) \leftarrow p(f(X))$, and then the subgoal $p(f(f(1)))$ is produced by resolution against the same clause. Setting $G \theta=p(f(f(1)))$, then its depth-3 abstraction, abs $(G \theta)$, is $p\left(f\left(f\left(X_{1.1 .1}\right)\right)\right)$ and by formula 2 , the subgoal $p\left(f\left(f\left(X_{1.1 .1}\right)\right)\right)$ would be called. This subgoal is completely evaluated producing the answers $p(f(f(0)))$ and $p(f(f(1)))$. After-

\footnotetext{
${ }^{10}$ See the XSB manual for the current list of properties with which depth- $k$ abstraction is compatible.
} 
wards, both solutions to $p\left(f\left(f\left(X_{1.1 .1}\right)\right)\right)$ would be post-unified with $p(f(f(1)))$ but would succeed only for $p(f(f(1)))$, which allows $X_{1.1 .1}$ to unify with 1 .

Implementation within the SLG-WAM. Our description of engine-level details of subgoal abstraction assumes some knowledge of the SLG-WAM engine, as presented in [Sagonas and Swift 1998; Ramakrishnan et al. 1999]. Let $G \theta$ be a tabled subgoal and assume that a maximum depth, $n$, has been set for the underlying predicate, $G_{P}$, of $G \theta$ and that the action is set to abstract. The abstraction is performed during the tabletry instruction (the SLG-WAM instruction corresponding to the NEW SUBGOAL operation). Within this instruction, a single-pass check-insert traversal of the subgoal $G \theta$ checks whether a variant of $G \theta$ has already been encountered during an evaluation, and creates a table for the subgoal if not. During this traversal, a depth counter is initialized by checking a cell in the table information frame for the predicate $G_{P}$; if the depth $n$ is reached at position $\pi_{k}$, a pointer to the subterm rooted at $\pi_{k}\left(\left.G \theta\right|_{\pi_{k}}\right)$ is added to an abstraction stack together with the (heap) address of $\pi_{k}$; then a free (position) variable $X_{\pi_{k}}$ is created at position $\pi_{k}$, and trailed with a pre-assignment cell in its trail frame, as used for mutable variables in XSB and other Prolog systems. Such a cell contains information about the value of a variable before a binding, and so supports backtrackable "destructive" assignment within a Prolog engine. After the abstraction and trailing, $X_{\pi_{k}}$ is copied to the table in the normal manner. If $G \theta$ is part of a set of mutually dependent subgoals, the SLG-WAM may need to repeatedly suspend and resume computation of $a b s(G \theta)$ as answers for other subgoals are derived and used for resolution. In general, the trail for the SLG-WAM supports suspending and resuming environments with a value cell: that is, it trails the value of the binding to a variable along with the variable itself. However, abstractions may also need to be undone and re-applied during environment switching; because pre-assignment trailing is used, the abstracted variables are reset to their prior (non-abstracted) terms when backtracking above the call to $G \theta$, then reset to their abstracted value via the value cell. To summarize, trail frames for abstracted variables require 1) the pre-assignment cell (in this case, pointing to $\left.G \theta\right|_{\pi_{k}}$ ); 2) the value cell (in this case, pointing to $X_{\pi_{k}}$ ); and 3) the variable address cell, just as in the WAM (in this case, the address of $X_{\pi_{k}}$ ). Trailing for non-abstracted variables does not require the pre-assignment cell ${ }^{11}$.

During the same check-insert traversal of the subgoal $G \theta$, the SLG-WAM creates a substitution factor: a vector that corresponds to the set of variables in $G \theta$. Substitution factors are maintained in the heap, and are not part of permanent table storage. The use of substitution factors allows the SLG-WAM to represent answers in a table as substitutions to the variables in a subgoal, which is more compact than representing answers as atoms that are instantiations of the subgoal [Ramakrishnan et al. 1999]. Using the substitution factor, when an answer is derived for a generator of $G \theta$ or returned to a consumer of $G \theta$, the engine need only copy bindings into or out of the table by traversing the substitution factor, rather than having to re-traverse the entire subgoal and answer. When subgoal abstraction is used for $G \theta$, the abstraction code ensures that the frames of the abstraction stack are also added to the substitution factor. The abstraction frames are then used for post-unification: the subterm in each abstracted position of the answer $\left(\left.a b s(G \theta) \eta\right|_{\pi_{k}}\right)$ is unified with the original subterm at that position $\left(\left.G \theta\right|_{\pi_{k}}\right)$. Only if all such unifications succeed is the answer return successful.

Example 5.2. Continuing from Example 5.1, for the abstracted subgoal $p\left(f\left(f\left(X_{1.1 .1}\right)\right)\right)$, the substitution factor would consist of the variable $X_{1.1 .1}$. When

\footnotetext{
${ }^{11}$ As noted in [Sagonas and Swift 1998] since the SLG-WAM trail represents a tree rather than a stack, frames also contain a cell that points to their previous trail frame. Such a cell is required regardless of whether a trailed variable has been abstracted.
} 
$\mathrm{p}_{-} 1(\mathrm{X}, \mathrm{F}):-\mathrm{q}_{-} 1(\mathrm{X})$.

P_2 $(X, F, Y):-$ q_2 $(X, Y)$.

$$
\begin{aligned}
& \text { p_1 }(X, F):-\operatorname{succ1mil}(X, Z), p_{-} 1(Z, F) . \\
& \text { p_2 }(X, F, Y):-\operatorname{succ1mil}(X, Z), p_{-} 2(Z, F, Y) .
\end{aligned}
$$

Fig. 3. Benchmark program.

evaluating $p\left(f\left(f\left(X_{1.1 .1}\right)\right)\right)$ the use of the substitution factor allows the engine to traverse only the bindings ( 0 and 1$)$ to $X_{1.1 .1}$ when copying answers into the table, and to store only these bindings: as indicated in [Ramakrishnan et al. 1999], no retraversal or storage of ancestor positions is necessary. In addition to the substitution

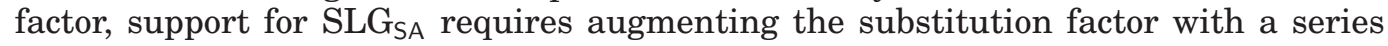
of abstraction frames, here a single frame containing $X_{1.1 .1}$ and 1 . When copying answers out of the table the variable $X_{1.1 .1}$ of the substitution factor is bound, and once this is accomplished, the post-unifications of the abstraction stack are performed: here unifying the bound value of $X_{1.1 .1}$ with 1 , so that only the binding $X_{1.1 .1}=1$ succeeds.

If a tabled subgoal contains attributed variables, the attributed variables are handled as follows. XSB tables subgoals with attributed variables by copying variable attributes into the table as specially designated terms. Suppose subgoal abstraction replaces a term $t$ rooted in position $\pi$ with a free variable $X_{\pi}$. If $t$ contains an attributed variable as a subterm, then the post-unification of the attributed variable may call a unification hook, just as any unification would, so that the abstraction code need not treat such abstracted variables in a special manner. However, if the depth bound is exceeded while traversing a variable attribute, abstraction is disabled until the attribute has been traversed. The reason for this is that abstracting midway through a variable attribute would break the unification hooks for many classes of attributes.

To summarize, engine-level implementation of subgoal abstraction first requires the ability to calculate the norm of a goal, and to apply an abstraction function. In the case of depth- $k$ abstraction, calculation of the norm and abstraction application can be done without an additional term traversal beyond that needed for tabling. Once the abstraction is performed, the abstraction vector and trail both maintain information on how to map the original term to the abstracted term. This information must be used whenever backtracking above the abstraction point or returning answers from the abstracted subgoal. While the implementation of subgoal abstraction demands care, the implementation of the above mechanisms: the depth check and abstraction, of abstraction vectors, and of the post-unification of abstracted answers with the original goal require a total of about 300-400 lines of code.

\subsection{Performance Overhead}

Subgoal abstraction obviously improves performance by ensuring termination when the atom dependency graph of a program contains an infinite path (as in $P_{f i n}$ of Example 1.1). Additionally, if an abstraction allows different subgoals to share the same table that otherwise would not, it can benefit performance in a similar manner as call subsumption. Because of these obvious benefits, its is natural to ask if there are cases when subgoal abstraction should not be used, a question we address here by measuring the performance overhead of subgoal abstraction.

To investigate the overhead of subgoal abstraction, a series of benchmark programs (Figure 3) were executed on a Macintosh laptop with a $2.43 \mathrm{GHz}$ Intel Core i5 processor and 4 GBytes of memory, running OS X 10.6.8. Timings on this platform show a variance of up to $6 \%$ for timings of the same executable and program. 
Table I. Benchmark results for tests of subgoal abstraction (times in seconds)

\begin{tabular}{lrrrrrrr} 
Program & 1 & $\mathrm{f}(1)$ & $\mathrm{f}^{2}(1)$ & $\mathrm{f}^{4}(1)$ & $\mathrm{f}^{8}(1)$ & $\mathrm{f}^{16}(1)$ & $\mathrm{f}^{32}(1)$ \\
\hline \hline p_1/2 (no answers) no abstr. & 0.424 & 0.471 & 0.517 & 0.693 & 0.96 & 1.214 & 1.702 \\
p_1/2 (no answers) abstr. & 0.431 & 0.489 & 0.533 & 0.72 & 0.864 & 0.862 & 0.864 \\
\hline p_1/2 $(1$ answer) no abstr & 0.524 & 0.576 & 0.62 & 0.808 & 1.071 & 1.324 & 1.841 \\
p_1/2 $(1$ answer) abstr & 0.529 & 0.579 & 0.621 & 0.809 & 1.009 & 1.008 & 1.01 \\
p_1/2 $(1$ answer + 4) no abstr & 0.534 & 0.583 & 0.623 & 0.809 & 1.072 & 1.332 & 1.839 \\
p_1/2 $(1$ answer + 4) abstr & 0.524 & 0.578 & 0.623 & 0.81 & 1.375 & 1.376 & 1.377 \\
p_1/2 $(1$ answer + 16) no abstr & 0.529 & 0.582 & 0.623 & 0.809 & 1.069 & 1.32 & 1.831 \\
p_1/2 $(1$ answer + 16) abstr & 0.525 & 0.58 & 0.625 & 0.809 & 2.336 & 2.341 & 2.352 \\
p_2/3 $(1$ answer) no abstr. & 0.633 & 0.676 & 0.765 & 0.928 & 1.147 & 1.398 & 1.917 \\
p_2/3 $(1$ answer) abstr. & 0.625 & 0.664 & 0.769 & 0.938 & 1.135 & 1.14 & 1.136 \\
p_2/3 $(4$ answers) no abstr. & 1.042 & 1.079 & 1.187 & 1.351 & 1.553 & 1.812 & 2.316 \\
p_2/3 (4 answers) abstr. & 1.024 & 1.067 & 1.168 & 1.334 & 1.651 & 1.658 & 1.649 \\
p_2/3 (8 answers) no abstr. & 1.485 & 1.535 & 1.625 & 1.795 & 2.016 & 2.256 & 2.768 \\
p_2/3 (8 answers) abstr. & 1.489 & 1.529 & 1.623 & 1.797 & 2.199 & 2.209 & 2.202
\end{tabular}

A first set of timings compared a version of XSB with subgoal abstraction implemented but not turned on, to the previous version without subgoal abstraction for various cases of linear recursion. These timings showed a difference in times well below the noise level. This is not surprising, as if subgoal abstraction is not invoked its only overhead is the maintenance of the depth counter during the check-insert step for tabled subgoals.

The next two series of timings test the overhead of subgoal abstraction when it is turned on, but does not provide an advantage in sharing tables for different subgoals. In these series, each benchmark makes use of the predicate succ1mil/2, the successor function for integers less than 1 million. The first series of benchmarks was constructed as follows.

- For $p_{\_} 1 / 2$ the base predicate $q_{-} 1 / 1$ was first set so that it never succeeded. Under this setting, the goal $p_{-} 1(0, F)$ creates 1 million variant subgoals, but no answers for any of these subgoals.

- Second, the fact $q_{-} 1(1000000)$ was added so that each of the million goals for $p_{-} 1 / 2$ contained a single answer.

- Next, 4 or 16 facts of the form $p_{-} 1\left(,{ }_{-}\right)$were also added. In these tests each subgoal had one answer that was redundantly rederived 4 or 16 times.

The second series of benchmarks was similar, but here the base predicate, $q \_$/ 2 , was adjusted so that 1,4 , or 8 answers was derived for each of 1 million subgoals. In this second series, no redundant answers were derived.

The benchmark series had two additional parameters. For each set of benchmarks the depth limit was either turned off or set to 6 . In addition, the top-level goals were $p_{-} 1(0, F)$ or $p_{\_} 2\left(0, F,{ }_{-}\right)$- where in each case $F$ was bound to terms of the form $f^{n}(1)$ for $n$ equal to $0,2,4,8,16$ and 32 (i.e., $f^{0}(1)=1, f^{1}(1)=f(1), f^{2}(1)=f(f(1))$, etc.). Note that the different values of $F$ do not affect the number of answers derived for any of these benchmark programs, but when $F$ was set to $f^{n}(1)$ for $n=8,16$, and 32 the subgoals are (non-trivially) abstracted if the depth-limit is set.

We first consider $p_{-} 1 / 2$. When no answers are derived, the timings for $p_{-} 1 / 2$ (Table I) show that subgoal abstraction reduces runtime up to $98 \%$ in the case of $f^{32}(1)$ compared to the runtime when subgoal abstraction is not used (cf. the first two lines of table Table I). In this case, if subgoal abstraction is not used, each goal needs to be fully traversed and copied into the table, but when subgoal abstraction is used, subterms with depth greater than $k$ do not need to be traversed: instead a pointer to the subterm is simply added to the abstraction stack, leading to efficiency for subgoal abstraction. As a contravening factor, if abstraction is not used, the $F$ argument is ground, while 
if abstraction is used the abstracted variable is added to the substitution factor, and must be traversed when copying an answer into or out of the table. Accordingly, when $p_{\_} 1 / 2$ derives a single answer per goal, subgoal abstraction still shows improvement above the noise level for $f^{16}(1)$ and $f^{32}(1)$. However as further redundant answers are derived the cost of traversing the binding to the redundant answer for the abstracted goal outweighs the savings made for the abstracted call, by up to $217 \%$ when 16 redundant answers are derived per goal. In the $p_{-} 1 / 2$ benchmarks, all answers but one per subgoal were redundant, so that scaling up to to extra answers per subgoal and to deeper bindings to $F$ measured only the cost of checking whether a given answer was already in the table. The post unification step was used only once, for the single nonredundant answer. On the other hand no answers for $p 2 / 2$ are redundant, so that in addition to the cost of answer check/insert, the cost of post-unification of each answer is also measured. Timings for $p \_2 / 3$ overall show less savings than those for $p_{-} 1 / 2$ for abstraction compared to non-abstraction; however the $p 2 / 3$ series still shows savings for $f^{16}(1)$ and $f^{32}(1)$.

These timings show that subgoal abstraction can be implemented so that its overhead is negligible if it is not used. If the number of answers per subgoal is relatively low and the subgoals are large, subgoal abstraction provides performance improvement by traversing the subgoal in almost a "lazy" manner. However, the cost for this is that the bindings for answers to abstracted goals will be larger than for non-abstracted goals, and traversing these answers to check for redundancy or to perform post-unification can lead to performance degradation, particularly when there are numerous answers per subgoal.

Comparisons with DLV. To our knowledge, the only other engine that is complete for (strongly) bounded term-size programs is that of DLV [Leone et al. 2002]. While XSB is an ISO-Prolog that supports tabling, DLV is an ASP system that has been extended to support function symbols [Alviano et al. 2010]. Despite their differences, the functionality of the two systems overlaps when computing queries to stratified programs, or grounding non-stratified programs. Repeated independent comparisons of DLV, XSB and other systems have been made in 2009, 2010 and 2011 by [OpenRuleBench 2011]. Although the last such comparison was performed in March 2011, the comparisons as a whole illustrate general performance differences between the two systems. XSB is generally faster and sometimes greatly faster than DLV for a variety of queries to definite programs including transitive closure, queries with stratified and non-stratified negation, and non-synthetic queries such as Wordnet ${ }^{12}$.

\section{DISCUSSION}

In this paper, we have examined the class of programs with canonical finite models and shown that it coincides with the class of strongly bounded term-size programs, whose definition is adapted from a well-known iterated fixed point definition of WFS [Przymusinski 1989]. Strongly bounded term-size programs in their turn, strictly include normal finitely ground programs, a class motivated by termination properties of grounders. The extension of SLG with the subgoal abstraction operation provides termination for queries to strongly bounded term-size programs so that the various decidable subclasses of finitely ground programs that have been identified in the literature can be used for analysis of termination for tabling systems as well as for ASP systems (e.g., [Syrjanen 2001; Gebser et al. 2007; Lierler and Lifshitz 2009; Eiter and Simkus 2009]. In addition, the query-orientation of SLGA makes it comparable with

\footnotetext{
${ }^{12}$ Exceptions are large tabled joins (where XSB consumes too much memory leading to thrashing), along with a puzzle example that includes function symbols.
} 
the technique of [Baselice and Bonatti 2010] on finitely recursive programs for which given queries may be strongly bounded term-size, but that may not have finitely representable models. SLG ${ }_{S A}$ has optimal complexity when using depth- $k$ abstractions (Theorem 4.19), and may produce a smaller program than other grounders (Theorem 4.16).

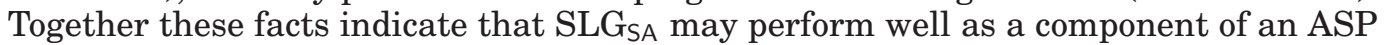
grounder. Finally, subgoal abstraction has been implemented at the engine level of XSB with good performance results in terms of overhead, query optimization, and comparison with other systems. The general approach presented here should be implementable without undue effort by other tabled Prologs, at least for definite programs.

Continued work with subgoal abstraction is needed to fully exploit its use in practical programs. One avenue for this is to explore abstraction methods other than depth$k$ : while depth- $k$ abstraction ensures termination and the best known complexity, the number of terms in $\mathcal{H}_{P}$ of $k$ or less can be large, even for small values of $k$. Accordingly the use of abstractions based on size or on a term-based weight should be explored, even if such abstractions are implemented by Prolog hooks, rather than within the engine itself. A second avenue is to support subgoal abstraction as a compiler optimization based on termination or cost analysis. Even without these features, however, subgoal abstraction increases the power of tabled logic programming for grounders, knowledge bases, and other applications.

\section{REFERENCES}

J.J. Alferes, M. Knorr, and T. Swift. 2013. Query-driven Procedures for Hybrid MKNF Knowledge Bases. ACM Transactions on Computational Logic 14, 2 (2013).

M. Alviano, W. Faber, and N. Leone. 2010. Disjunctive ASP with Functions: Decidable Queries and Effective Computation. Theory and Practicce of Logic Programming 10, 4-6 (2010), 497-512.

S. Baselice and P. Bonatti. 2010. A decidable subclass of finitary programs. Theory and Practicce of Logic Programming 10, 4-6 (2010), 481-496.

S. Baselice, P. Bonatti, and G. Criscuolo. 2009. On finitely recursive programs. Theory and Practice of Logic Programming 9, 2 (2009), 213-238.

F. Calimeri, S. Cozza, G. Ianni, and N. Leone. 2008. Computable Functions in ASP: Theory and Implementation. In International Conference on Logic Programming (LNCS), Vol. 5366. Springer, 407-424.

W. Chen and D. S. Warren. 1996. Tabled Evaluation with Delaying for General Logic Programs. Journal of the Association for Computing Machinery 43, 1 (1996), 20-74.

S. Decorte, D. De Schreye, and H. Vandecasteele. 1999. Constraint-based Termination Analysis of Logic Programs. ACM Transactions on Programming Languages and Systems 21 (1999), 1137-1195.

T. Eiter and M. Šimkus. 2009. FDNC: Decidable Nonmontonic Disjunctive Logic Progrmas with Function Symbols. ACM Transactions on Computational Logic 9, 9 (2009), 1-45.

M. Gebser, T. Schaub, and S. Thiele. 2007. GrinGo: A New Grounder for Answer Set Programs. In Logic Programming and Non-Monotonic Reasoning. 267-280.

M. Gelfond and V. Lifschitz. 1988. The Stable Model Semantics for Logic Programming. In International Conference and Symposium on Logic Programming. 1070-1080.

B. Grosof, M. Dean, and M. Kifer. 2012. Semantic Web Rules: Fundamentals, Applications, and Standards. (2012). Tutorial, 11th International Semantic Web Conference.

N. Leone, G. Pfeifer, W. Faber, F. Calimeri, T. Dell'Armi, T. Eiter, G. Gottlob, G. Ianni, G. Ielpa, K. Koch, S. Perri, and A. Polleres. 2002. The DLV system. In JELIA. 537-540.

Y. Lierler and V. Lifshitz. 2009. One more decidable class of finitely ground progrmas. In International Conference on Logic Programming.

J. W. Lloyd. 1987. Foundations of Logic Programming (2nd extended ed.). Springer-Verlag.

OpenRuleBench. 2009-2011. OpenRuleBench: Benchmarks for Semantic Web Rule Engines. (2009-2011). $\backslash$ sfrulebench.projects.semwebcentral.org

T. Przymusinski. 1989. Every Logic Program has a Natural Stratification and an Iterated Least Fixed Point Model. In ACM Symposium on Principles of Database Systems. 11-21.

I. V. Ramakrishnan, P. Rao, K. Sagonas, T. Swift, and D. S. Warren. 1999. Efficient Access Mechanisms for Tabled Logic Programs. Journal of Logic Programming 38, 1 (1999), 31-55. 
F. Riguzzi and T. Swift. 2013. Well-Definedness and Efficient Inference for Probabilistic Logic Programming under the Distribution Semantics. (2013). To appear in Theory and Practice of Logic Programming. Available at journals.cambridge.org/article_S1471068411000664.

K. Sagonas and T. Swift. 1998. An Abstract Machine for Tabled Execution of Fixed-Order Stratified Logic Programs. ACM Transactions on Programming Languages and Systems 20, 3 (May 1998), 586 - 635.

T. Swift. 1999. A New Formulation of Tabled Resolution with Delay. In Recent Advances in Artifiial Intelligence (LNAI), Vol. 1695. Springer, 163-177.

T. Swift and D.S. Warren. 2012. XSB: Extending the Power of Prolog using Tabling. Theory and Practicce of Logic Programming 12, 1-2 (2012), 157-187.

T. Syrjanen. 2001. Omega-Restricted Logic Programs. In Logic Programming and Non-Monotonic Reasoning. 267-280.

H. Tamaki and T. Sato. 1986. OLDT Resolution with Tabulation. In International Conference on Logic Programming (LNCS), Vol. 225. Springer, 84-98.

A. van Gelder. 1989. The Alternating Fixpoint of Logic Programs with Negation. In ACM Symposium on Principles of Database Systems. 1-10.

A. van Gelder, K. A. Ross, and J. S. Schlipf. 1991. The Well-founded Semantics for General Logic Programs. Journal of the Association for Computing Machinery 38, 3 (1991), 620-650.

S. Verbaeten, D. De Schreye, and K. Sagonas. 2001. Termination proofs for logic programs with tabling. ACM Transactions on Computational Logic 2, 1 (2001), 57-92.

D. Voets and D. De Schreye. 2011. Non-termination analysis of logic programs with integer arithmetics. Theory and Practicce of Logic Programming 11, 4-5 (2011), 521-536.

G. Yang, M. Kifer, H. Wan, and C. Zhao. 2012. FLORA-2: User's Manual Version 0.97. 


\section{A. LONGER PROOFS}

\section{A.1. Proofs for Results of Section 4.4 (Correctness and Termination of SLG SA $_{\text {) }}$}

THEOREM 4.13 Let $\mathcal{E}$ be an $S L G_{\mathrm{SA}}$ evaluation of a query $Q$ to a safe program $P$. Then $\mathcal{I}_{\mathcal{F}_{\text {fin }}}=\left.W F M^{P}\right|_{\mathcal{F}_{\text {fin }}}$.

PROOF. Note that the only difference between SLGA and the version of SLG from Section 4.2 is that in SLG $_{S A}$ the root subgoals of some trees in a forest $\mathcal{F} \in \mathcal{E}$ may have been abstracted. We consider the two cases in which an abstraction operation may be used and show that the action of the abstraction function is the same as a simple rewriting of a rule that preserves logical equivalence, but can be evaluated by SLG without an abstraction function. The theorem then holds by the correctness of SLG (cf. [Chen and Warren 1996], and [Swift 1999] for the forest of trees model).

- Positive selected literals. Let $N=(G \leftarrow$ Delays $\mid$ Goals $) \theta$ be a node with selected positive literal $S$. Note that $N$ must have an ancestor in the tree for $G$ that was created by a program clause

$$
r=H \leftarrow A_{1}, \ldots, A, \ldots, L_{n}
$$

and assume that $S$ corresponds to the selected literal $A$ so that $S=A \theta$. Suppose that a NEW SUBGOAL operation creates a tree with root subgoal $A^{\prime}=a b s(A \theta)$. We have that $A \theta=A^{\prime} \eta$ for some $\eta$, as $A^{\prime}$ subsumes $A \theta$.

Let

$$
r^{\prime}=\left(H \leftarrow A_{1}, \ldots, \operatorname{abs}\left(A, A^{\prime}\right), A^{\prime}, A^{\prime}=A, \ldots, L_{n}\right)
$$

The predicate abs/2 simply sets $A^{\prime}=a b s(A)$ (its implementation is outside of the semantics of the SLG evaluation $\mathcal{E}$ ). $A^{\prime}$ is then called, and if it succeeds, $A^{\prime}$ is unified with $A$. Note that $r^{\prime}$ is logically equivalent to $r$ : $A^{\prime}$ subsumes $A$ so that every solution to $A$ will also be a solution to $A^{\prime}$, and the unification $A^{\prime}=A$ in $r^{\prime}$ ensures that only those solutions that also unify with $A$ will succeed.

- Negative selected literals. The argument is essentially the same, but the transformation is instead:

$$
r^{\prime}=\left(H \leftarrow A_{1}, \ldots, a b s\left(A, A^{\prime}\right), \text { not exists_ans }\left(A^{\prime}, A\right), \ldots, L_{n}\right)
$$

where

$$
\text { exists_ans }\left(A^{\prime}, A\right) \leftarrow A^{\prime}, A=A^{\prime} .
$$

By assumption, $P$ is safe, so that $A^{\prime}$ is ground when the literal $A^{\prime}=A$ is called. Note that not exists_ans $\left(A^{\prime}, A\right)$ succeeds iff there is no (ground) answer to the goal $A^{\prime}$ that unifies with the ground $A$. As above, the program $r^{\prime}$ is logically equivalent to $r$.

The program $P^{\prime}$ is constructed from $P$ by the transformations of the various rules (sometimes replacing a rule by a series of transformations), and adding $a b s / 2$ and exists_ans $/ 2$. Note that the transformation is completely local to a rule, so that replacing all rules in $P$ by forms equivalent to $r^{\prime}$ makes $P^{\prime}$ a conservative extension of $P$.

THEOREM 4.14 Let $Q$ be a query to a strongly bounded term-size program $P$. Then any $S L G_{\mathrm{SA}}$ evaluation $\mathcal{E}$ of $Q$ that uses a finitary abstraction operation reaches a final forest $\mathcal{F}_{\text {fin }}$ after a finite number of steps. If $P$ is safe, then $\mathcal{F}_{\text {fin }}$ will not be floundered.

PROOF. We first prove the statement that any SLG evaluation $\mathcal{E}$ of $Q$ that uses a finitary abstraction operation reaches a final forest $\mathcal{F}_{\text {fin }}$ after a finite number of steps. The proof is by induction on the maximal dynamic stratum of any answer in $\mathcal{E}$.

- For the base case, assume the maximal stratum is 1 (Definition 2.2). Because $Q$ is in stratum 1 , the only applicable SLG operations in $\mathcal{E}$ are NEW SUBGOAL, ProGram 
Clause Resolution, Positive Return, and Completion. Note that each of these operations is applicable only once to a given node or set of subgoals.

- NEW SUBGOAL: The use of a finitary abstraction operation means that there may only be a finite number of NEW SUBGOAL operations in $\mathcal{E}$, and hence a finite number of trees in any forest of $\mathcal{E}$.

- Program Clause Resolution: Since there are a finite number of trees, and a finite number of program clauses resolvable against the root subgoal of any tree, $\mathcal{E}$ contains only a finite number of PROGRAM ClaUSE RESOLUTION operations, and the root of any tree has only a finite number of immediate children.

- Positive Return: Next, since $P$ is strongly bounded term-size, true $\left(W F M^{P}\right)$ is finite, and since the maximal stratum of $\mathcal{E}$ is 1 , any answer returned will be unconditional. Accordingly there are a finite number of answers that can be resolved against any selected subgoal. Because interior nodes of SLG trees can only be extended by POSITIVE RETURN operations, any non-root node in any tree may have only a finite number of children. In addition, the depth of any tree in $\mathcal{E}$ is bounded by the maximal number of body literals in any rule in $P$, which is finite. Thus the subtrees of any tree in $\mathcal{E}$ have a finite depth and a finite branching factor, and so are finite. There can thus be only a finite number of POSITIVE RETURN operations.

- COMPLETION: Finally, since there are a finite number of trees in any forest, there can be only a finite number of COMPLETION operations.

Since the number of occurrences of each type of operation in $\mathcal{E}$ is finite, $\mathcal{E}$ itself must be finite.

- For the inductive case, assume that the statement is true for all atoms whose (finite) stratum is less than $n$ in order to prove it true for those atoms whose stratum is $n$.

- New Subgoal, Program Clause Resolution and Completion are argued in the same manner as for the base case.

- Positive Return: Because $P$ is strongly bounded term-size there are only a finite number of undefined atoms. Since each literal in the Delays of a node must come from delaying or SLG resolution of a literal in the body of a rule, and the maximum number of literals in the body of a rule is finite, there are only a finite number of conditional answers. The statement that there are only a finite number of POSITIVE RETURN operations follows as for the base case.

- NEGATIVe RETURN: First, consider that a NEGATIVE RETURN operation can be applied at most once to any node $N$. As a result of this operation, any node $N$ to which a NEGATIVE RETURN operation is applied can have only a single child: either a failure node in the case of NEGATION FAILURE, or a single child with the selected literal removed from the Goals of $N$ in the case of NEGATION SUCCESS. In the case of NEGATION FAILURE this is enough to show that the finiteness of $\mathcal{E}$ is not affected, as a failure node cannot be further expanded. In the case of NEGATION SuCCESS, the fact that the selected literal is removed from Goals, means that the child of $N$ will have a smaller Goals sequence. Since Goals is finite, any path from $N$ may have only a finite number of NEGATIVE RETURN operations.

- DELAYING: Considerations analogous to the NEGATiOn SuCCESS case show that DELAYING will not affect the finiteness of $\mathcal{E}$.

- Answer Completion: Next, note that Answer Completion will produce a single failure node as a child of each answer node to which it is applied, and a failure node cannot be further expanded. So ANSWER COMPLETION does not affect the finiteness of any forest, no matter how many times it is applied.

- In the case of SimplificATION, an application of the SimplificATION operation that produces a failure node does not affect the finiteness of any forest (Defini- 
tion $4.6,6 a$ ) as a failure node cannot be further expanded.. On the other hand, if an application of a SIMPLIFICATION operation to a node $N$ produces a non-failure child (Definition 4.6,6b), note that similar to the case of NEGATION SUCCESS, the child of $N$ will have a smaller Delays. Since Delays is finite, any path from $N$ to its descendents may have only a finite number of SIMPLIFICATION operations. Since each operation can be applied only a finite number of times, $\mathcal{E}$ must be finite.

Note that the proof of the previous statement shows that $\mathcal{E}$ is finite, but $\mathcal{F}_{\text {fin }}$ may be floundered: i.e., a node in $\mathcal{F}_{\text {fin }}$ may have a selected non-ground negative literal. We next show that if $P$ is safe, then $\mathcal{F}_{\text {fin }}$ will not be floundered. It is straightforward to show that if $P$ is safe, any answer in any forest in $\mathcal{E}$ will be ground. Then, let $r$ be a rule in a safe program $P$, and $L_{j}$ a given negative literal in $r$. Because of the safety of $P$, the action of Program Clause Resolution and Positive Return operations on previously selected subgoals to the left of $L_{j}$ in $r$ will ensure that $L_{j}$ is ground by the time it becomes a selected subgoal.

\section{A.2. Proofs for Results of Section 4.5 (Comparison with Intelligent Instantiation)}

THEOREM 4.16

Let $P$ be a safe, finitely ground program $P$. Let $\mathcal{E}$ be a SLGA evaluation of a grounding predicate of $P$ whose final forest is $\mathcal{F}_{\text {fin }}$, and $P_{\text {tabled }}=\operatorname{answers}\left(\mathcal{F}_{\text {fin }}\right)$.

(1) $P_{\text {tabled }}$ is equal to $\frac{\operatorname{ground}(P)}{W F M^{P}}$.

(2) Let $P_{i i}$ be the intelligent instantiation of $P$. Then $P_{\text {tabled }} \geq_{\text {red }} P_{i i}$.

Proof.

(1) (Sketch) We consider first the case of SLG (i.e., where no abstraction operation is used). By the correctness of SLG, the use of the grounding predicate ensures that $\mathcal{I}_{\mathcal{F}_{\text {fin }}}=W F M^{P}$. The safety of $P$ ensures that all answers are ground, regardless of whether they are conditional. In such a case, the correctness of SLG with respect to the stable model semantics (cf. [Chen and Warren 1996]), ensures that if there is rule $r$ with a non-empty body in $\frac{\text { ground }(P)}{W F M^{P}}$, then $r$ must be a conditional answer in $\mathcal{F}_{\text {fin }}$.

For SLGA

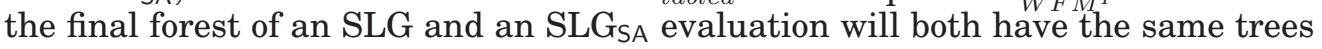
for subgoals immediatedly called by the grounding predicate (which we call here the grounding subgoals), and the answers in each of these trees will be the same. For other trees, suppose that the $\mathrm{SLG}_{\mathrm{SA}} \mathcal{E}_{\mathrm{SA}}$ evaluation differed from the SLG evaluation $\mathcal{E}$ in that $\mathcal{E}_{\mathrm{SA}}$ created a tree for an abstracted subgoal, while $\mathcal{E}$ did not. Both of these subgoals are more specific than the grounding subgoals, and the (ground) answers of both are contained in the set of ground answers for the grounding subgoals.

(2) Follows immediately from Lemma 2.26 and part 1 of this theorem.

\section{A.3. Proofs for Complexity Results of Section 4.6}

LEMMA 4.18. Let $P$ be a ground program, $Q$ a ground query, and $\mathcal{E}$ a terminating $S L G_{\mathrm{SA}}$ evaluation of $Q$ against $P$ that uses depth-k abstraction. Then the number of nodes in the final forest $\mathcal{F}_{\text {fin }}$ is at most $\mathcal{O}\left(\operatorname{size}\left(P_{Q}(\mathcal{E})\right)\right)$..

PROOF. First note that since no step of an SLG $_{S A}$ evaluation ever removes a node or tree from a forest, showing the upper bound for $\mathcal{F}_{\text {fin }}$ carries over to all forests in 
$\mathcal{E}$. We consider first the special case in which $Q$ (finitely) terminates using the identity function as an abstraction function (i.e., depth- $\omega$ abstraction), before the case of general abstraction functions.

(1) $a b s(\cdot)$ is the identity function. Within this case, we consider first the subcase where $P$ is definite, and then consider the case where $P$ is any normal program.

$-P$ is definite. First, since $P$ is ground, no two trees in any forest of $\mathcal{E}$ may have root subgoals that unify. Because of this fact, each rule in $P_{Q}$ may be used for resolution in at most one tree in $\mathcal{F}_{\text {fin }}$. Continuing, consider a rule $r$ of $P_{Q}$ that creates a node $n_{r}$ in some tree in $\mathcal{F}_{\text {fin }}$. The number of descendants of $n_{r}$ is at most the number of literals in the body of $r$. To see this, first note that the selected literal of any node is ground and thus may have at most once descendant. Next, since each POSITIVE RETURN answer operation removes a body literal from the Goals of a node when creating a new node, the number of descendants of $n$ is limited to the number of body literals in $n_{r}$. Thus there are at most size $\left(P_{Q}\right)$ non-root nodes in $\mathcal{F}_{\text {fin }}$ and $\mathcal{O}\left(\operatorname{size}\left(P_{Q}(\mathcal{E})\right)\right)$ nodes overall.

$-P$ is a normal program. If $P$ is not definite, then a node $n_{r}$ as above may have at most $2 \times \operatorname{size}(r)$ descendents, as each body literal first may be delayed and then either simplified or failed via an ANSWER Completion operation. So in this case there are still $\mathcal{O}\left(\operatorname{size}\left(P_{Q}(\mathcal{E})\right)\right)$ nodes overall.

(2) abs $(\cdot)$ is a depth-k abstraction function for positive integer $k$. To show this result,we first show a subsidiary statement.

Consider two subgoals abs $\left(S_{1}\right)$ and $a b s\left(S_{2}\right)$ at the root of two distinct trees in $\mathcal{F}_{\text {fin }}$ generated from the application of NEW SUBGOAL to $S_{1}$ and $S_{2}$. We show that abs $\left(S_{1}\right)$ cannot unify with $a b s\left(S_{2}\right)$. Recall that $a b s\left(S_{1}\right)$ and $a b s\left(S_{2}\right)$ cannot be identical (up to variance), since they would not correspond to distinct trees in that case. There are three cases to consider.

(a) abs $\left(S_{1}\right)=S_{1}$ and $a b s\left(S_{2}\right)=S_{2}$. Since $P$ is ground, this means that $S_{1}$ and $S_{2}$ are ground, so $a b s\left(S_{1}\right)$ does not unify with abs $\left(S_{2}\right)$.

(b) $\operatorname{abs}\left(S_{1}\right)=S_{1}$ but $a b s\left(S_{2}\right) \neq S_{2}$. In this case, there must be a position $\pi$ in $S_{2}$ in which a constant or function symbol was replaced by a position variable. However, $S_{1}$ is ground since $P$ is ground, but $S_{1}$ does not have a position of depth $k$ as abs $\left(S_{1}\right)=S_{1}$. Accordingly there must be some position $\pi^{\prime}$ that is a constant in $a b s\left(S_{1}\right)$, but is a non-constant function symbol in $a b s\left(S_{2}\right)$, so that $a b s\left(S_{1}\right)$ and $a b s\left(S_{2}\right)$ do not unify.

(c) $a b s\left(S_{1}\right) \neq S_{1}$ and $a b s\left(S_{2}\right) \neq S_{2}$. In this case,

i. Either there is some position $\pi$ in one of the subgoals, say abs $\left(S_{1}\right)$ that is not a position variable, while $\operatorname{abs}\left(S_{2}\right)$ has a position variable at $\pi$. For this subcase the argument is identical to case 2(b) above.

ii. Otherwise if $a b s\left(S_{1}\right)$ and $a b s\left(S_{2}\right)$ contain position variables in exactly the same positions, recall that $a b s\left(S_{1}\right)$ and $a b s\left(S_{2}\right)$ cannot be identical (variants) of each other, so there must be some position $\pi$ in which abs $\left(S_{1}\right)$ differs from $a b s\left(S_{2}\right)$ and neither $\left.a b s\left(S_{1}\right)\right|_{\pi}$ nor $\left.a b s\left(S_{2}\right)\right|_{\pi}$ is a position variable. Since $P$ is ground, abs $\left.\left(S_{1}\right)\right|_{\pi}$ cannot unify with $\left.a b s\left(S_{2}\right)\right|_{\pi}$

Since no two subgoals for trees in any forest $\mathcal{F}$ of $\mathcal{E}$ may unify and since $P$ is ground, each program clause of $P_{Q}$ can appear in at most one tree in $\mathcal{F}$. Given this fact the argument of case 1 can be applied when depth- $k$ abstraction is performed for some integer $k>0$. 
THEOREM 4.19. Let $P$ be a ground program, $Q$ a ground query, and $\mathcal{E}$ a terminating $S L G_{\mathrm{SA}}$ evaluation of $Q$ against $P$ that uses depth-k abstraction. Then under the cost model $\mathcal{C}_{\text {function, }}$ the cost of $\mathcal{E}$ is $\mathcal{O}\left(\mid\right.$ atoms $\left.\left.\left(\mathcal{F}_{\text {fin }}\right)\right) \mid \times \operatorname{size}\left(P_{Q}(\mathcal{E})\right)\right)$.

PROOF. From Lemma 4.18 there are $\mathcal{O}\left(\operatorname{size}\left(P_{Q}(\mathcal{E})\right)\right)$ nodes in $\mathcal{F}_{\text {fin }}$. First note that

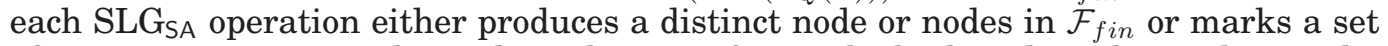
of trees in $\mathcal{F}_{\text {fin }}$ as complete. Thus, the cost of $\mathcal{E}$ can be broken down by analyzing the costs of creating distinct set of nodes.

- New Subgoal, Program Clause Resolution, Positive Return, Negative Return, Delaying, Simplification. Since each of these operations is constant-time in $\mathcal{C}_{\text {function }}$, they produce a node in $\mathcal{F}_{\text {fin }}$ and there can be at most $\mathcal{O}\left(\operatorname{size}\left(P_{Q}(\mathcal{E})\right)\right)$ such operations, the combined cost of these operations is $\mathcal{O}\left(\operatorname{size}\left(P_{Q}(\mathcal{E})\right)\right)$.

- COMPLETION. The cost of the COMPLETION operation is non-constant; rather, its cost is the number of trees it marks as complete. Since each tree is marked as completed only once, the combined cost of the COMPLETION operations is at most $\left|\operatorname{atoms}\left(\mathcal{F}_{\text {fin }}\right)\right|$.

- Answer Completion. The cost of the Answer Completion operation is again non-constant. The cost of an ANSWER COMPLETION operation is at most size $\left(P_{Q}(\mathcal{E})\right)$, however each ANSWER COMPLETION operation must produce failure nodes for all answers whose head is some unsupported atom $A$. Afterwards, $A$ will no longer be subject to an ANSWER COMPLETION operation. Since $P$ is ground, $A$ corresponds to an element of atoms $\left.\left(\mathcal{F}_{\text {fin }}\right)\right)$. Thus there are thus at most $\left.\mid \operatorname{atoms}\left(\mathcal{F}_{\text {fin }}\right)\right) \mid$ ANSWER COMPLETION operations.

The total worst-case cost of $\mathcal{E}$ is thus the cost of the constant time operations $\mathcal{O}\left(\operatorname{size}\left(P_{Q}(\mathcal{E})\right)\right)$ plus the cost of the COMPLETION operations $\mathcal{O}\left(\left|a t o m s\left(\mathcal{F}_{\text {fin }}\right)\right|\right)$, plus the cost of the ANSWER COMPLETION operations $\mathcal{O}\left(\left|a t o m s\left(\mathcal{F}_{\text {fin }}\right)\right| \times \operatorname{size}\left(P_{Q}(\mathcal{E})\right)\right)$ so that the total cost of $\mathcal{E}$ is $\mathcal{O}\left(\left|\operatorname{atoms}\left(\mathcal{F}_{\text {fin }}\right)\right| \times \operatorname{size}\left(P_{Q}(\mathcal{E})\right)\right)$. 